ARTICLE

https://doi.org/10.1038/s41467-021-26535-x

\title{
OPEN
}

\section{Structural basis for high selectivity of a rice silicon channel Lsi1}

Yasunori Saitoh (10 1,7, Namiki Mitani-Ueno 2,7 , Keisuke Saito (1) 3,4,7, Kengo Matsuki (D) ${ }^{5}$, Sheng Huang ${ }^{2}$,

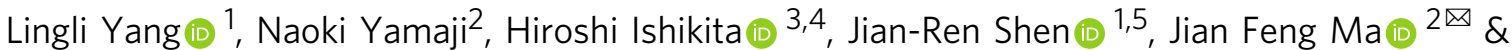
Michihiro Suga (iD) 1,5,6凶

Silicon ( $\mathrm{Si}$ ), the most abundant mineral element in the earth's crust, is taken up by plant roots in the form of silicic acid through Low silicon rice 1 (Lsi1). Lsi1 belongs to the Nodulin 26-like intrinsic protein subfamily in aquaporin and shows high selectivity for silicic acid. To uncover the structural basis for this high selectivity, here we show the crystal structure of the rice Lsi1 at a resolution of $1.8 \AA$. The structure reveals transmembrane helical orientations different from other aquaporins, characterized by a unique, widely opened, and hydrophilic selectivity filter (SF) composed of five residues. Our structural, functional, and theoretical investigations provide a solid structural basis for the Si uptake mechanism in plants, which will contribute to secure and sustainable rice production by manipulating Lsi1 selectivity for different metalloids.

\footnotetext{
${ }^{1}$ Research Institute for Interdisciplinary Science, Okayama University, Tsushima Naka 3-1-1, Kita, Okayama 700-8530, Japan. ${ }^{2}$ Institute of Plant Science and Resources, Okayama University, Chuo 2-20-1, Kurashiki 710-0046, Japan. ${ }^{3}$ Research Center for Advanced Science and Technology, The University of Tokyo, 4-6-1 Komaba, Meguro-Ku, Tokyo 153-8904, Japan. ${ }^{4}$ Department of Applied Chemistry, Graduate School of Engineering, The University of Tokyo, 7-3-1 Hongo, Bunkyo-Ku, Tokyo 113-8654, Japan. ${ }^{5}$ Graduate School of Natural Science and Technology, Okayama University, 3-1-1 Tsushima Naka, Okayama $700-$ 8530, Japan. ${ }^{6}$ Japan Science and Technology Agency, PRESTO, 4-1-8 Honcho, Kawaguchi, Saitama 332-0012, Japan. ${ }^{7}$ These authors contributed equally: Yasunori Saitoh, Namiki Mitani-Ueno, Keisuke Saito. 凶email: maj@rib.okayama-u.ac.jp; michisuga@okayama-u.ac.jp
} 
A 11 plants rooting in soil contain a significant amount of silicon ( $\mathrm{Si}$ ) in their bodies ${ }^{1-3}$. Although $\mathrm{Si}$ has not been recognized as an essential element for plant growth, its beneficial effects have been observed in many plant species. Si is especially essential for the high and stable production of rice (Oryza sativa), which is able to accumulate $\mathrm{Si}$ in the shoots to up to $10 \% \mathrm{Si}$ of dry weight ${ }^{3}$. This high $\mathrm{Si}$ accumulation helps plants to overcome various biotic (e.g., pest, disease) and abiotic (e.g., lodging, nutrient imbalance, metal toxicity) stresses ${ }^{1-4}$. Due to its importance in rice production, $\mathrm{Si}$ has been recognized as an "agronomically essential element", in Japan and Si fertilizers are routinely applied to the paddy field ${ }^{3}$.

Plant roots take up Si from soil solution as silicic acid $\mathrm{Si}(\mathrm{OH})_{4}$, an uncharged monomeric molecule at a $\mathrm{pH}$ below 9. High accumulation of $\mathrm{Si}$ in rice shoots is achieved by the cooperation of two different transporters for silicic acid; Low Si rice 1 and 2 (Lsi1 and Lsi 2$)^{5-7}$. Lsi1 and Lsi2 are polarly localized at the distal and proximal sides of both exodermis and endodermis of the roots, respectively, and are responsible for Si uptake ${ }^{5-7}$. Knockout of either Lsi1 or Lsi2 results in a significant decrease in Si uptake and rice grain yield ${ }^{5-7}$. Lsil belongs to the Nodulin 26-like intrinsic proteins (NIPs) subfamily in the aquaporin (AQP) family ${ }^{6}$, while Lsi2 belongs to the ion transporter superfamily ${ }^{7}$. NIPs are unique members of AQP because they are only present in plants but not in animals. Furthermore, among NIPs, only a small group (NIP III), including Lsi1, shows transport activity for silicic acid ${ }^{8-10}$, while other members transport boric acid, arsenite, and glycerol $^{11,12}$ (Fig. 1a and Supplementary Table 1). These findings indicate that Lsil has a distinct selectivity for silicic acid, but the structural basis for this high selectivity is unknown.

In the present study, we show the crystal structure of rice Lsi1 at $1.8 \AA$ resolution and compare it with that of water-specific $\mathrm{AQP} 1^{13}$ and glycerol permeable aquaglyceroporin $\mathrm{GlpF}^{14}$, and other known AQP structures. The structure of Lsil reveals unique transmembrane (TM) helical orientations, the selectivity filter (SF), and water molecules in the channel that are distinct from the other structurally characterized AQPs. Mutational studies based on the high-resolution structure and theoretical calculations uncover the principles of silicic acid permeability.

\section{Results}

The overall structure of Lsi1. To obtain the crystal of Lsi1, we used fluorescence-detection size-exclusion chromatography $(\text { FSEC })^{15}$. However, we failed to obtain crystals of full-length Lsil, so we screened a large variety of mutant Lsil and found that a mutant starting at Leu47 and ending at Arg264 ( $\Delta \mathrm{N} 44 / \Delta \mathrm{C} 24 /$ K50R/C66A/T93V/C139A/K232R/T253V/K264R) gave rise to crystals (Supplementary Figs. 1 and 2). This Lsi1 variant, Lsi $1_{\text {cryst }}$, is functional in transporting $\mathrm{Ge}(\mathrm{Si}$ analog) based on assay in Sf9 insect cells (Supplementary Fig. 3). Compared to the C-terminally EGFP-tagged full-length Lsil (CE-Lsi1), both CE-Lsil $1_{\text {cryst }}$ and the $\mathrm{N}$ - and C- terminally truncated constructs (CE-Lsi1_ $\Delta \mathrm{NC}$ ) showed a slightly reduced Ge transport activity (about $60 \%$ of wild type) (Supplementary Fig. 3). Crystals of Lsil $1_{\text {cryst }}$ diffracted to $1.6 \AA$ resolution but suffered from lattice-translocation defects $^{16}$. We corrected X-ray intensities with the approach of Wang et al. ${ }^{16}$ and solved the structure of Lsil $1_{\text {cryst }}$ at $1.8 \AA$ resolution (Table 1). The structure of Lsi $1_{\text {cryst }}$ reveals a tetrameric fold similar to other AQPs from bacteria ${ }^{14}$, plants ${ }^{17,18}$, and animals ${ }^{13,19}$. Each monomer contains six transmembrane helices (TM1-TM6), five connecting loops (loop A- loop E), and two half helices ( $\mathrm{HB}$ and $\mathrm{HE}$ ) with $\mathrm{N}$ and $\mathrm{C}$-terminus located on the cytoplasmic side of the membrane (Fig. 1b, c). The highresolution electron density map allowed us to build all side-chain residues (Ala46 through Arg264) unambiguously. About 120 water molecules per monomer were also identified in the highresolution structure (Figs. 1d and 2e). The channel pore exists in each monomer with a constriction on the extracellular side, similar to the other AQP structures ${ }^{13,14,17}$.

However, there are notable differences between water-specific AQPs and aquaglyceroporins in the loop regions and the tilting of TM helices. Compared to other AQP structures, including AQP1 ${ }^{13}$ (PDB 1J4N) and $\mathrm{GlpF}^{14}$ (PDB 1FX8), Lsil $1_{\text {cryst }}$ has a shift in TM1, TM4, TM5, and HE at the extracellular side towards the center of the channel, whereas its TM2 shifts towards a pseudo- $c 4$ axis of the tetramer (Fig. 2a, b and Supplementary Fig. 4). A few residues unique to the $\mathrm{Si}$ permeable AQPs (Gly88 ${ }_{\mathrm{TM} 2}$, Val173, Thr206, Ser207 ${ }_{\mathrm{TM} 5}$, and Gly216 ${ }_{\mathrm{LE} 1}$ ) can explain such shifts well. AQP1 has bulky residues Phe $58_{\mathrm{TM} 2}$ and His $182_{\mathrm{TM}}$ in SF, whereas they are replaced by smaller ones in Lsi $_{\text {cryst, }}$ Gly88 $8_{\mathrm{TM} 2}$, and Ser207 ${ }_{\mathrm{TM} 5}$ (Fig. 3a, b). Two bulky residues in SF of GlpF, Trp48 ${ }_{\mathrm{TM} 2}$, and Phe200 2 LE1, are also replaced by Gly residues in Lsi $1_{\text {cryst }}$, Gly $88_{\mathrm{TM} 2}$, and Gly216 $6_{\mathrm{LE} 1}$ (Fig. 3a, c). The smaller residues in $\mathrm{Lsi1}_{\text {cryst }}\left(\mathrm{Gly}_{\mathrm{T}} 8_{\mathrm{TM} 2}\right.$, Ser207 $7_{\mathrm{TM} 5}$, and Gly216 $\mathrm{LE}_{\mathrm{LE}}$ ) alleviate steric restriction and allow the shifts of the TM1, TM2, TM5, and HE. In addition, extracellular loop A of Lsil $1_{\text {cryst }}$ (Gly73 to Ser80) is shorter than other AQPs, which may be related to the close approach of TM1 and TM2. AQP1 has Gly147 in TM4 and Gly181 in TM5, making a close contact between TM4 and TM5 possible, whereas the equivalent residues in $\mathrm{Lsil}_{\text {cryst }}$ are Val173 and Thr206, which disable the close approach in the center of the bilayer region. Instead, loop C interacts with the tips of TM4 and TM5, assisting

\section{Table 1 Crystallographic data collection and refinement statistics.}

\section{Data collection}

\begin{tabular}{|c|c|}
\hline Wavelength $(\AA)$ & 1.112181 \\
\hline Space group & $P 12,1$ \\
\hline Cell dimensions & $\begin{array}{l}a=89.5 \AA, b=91.4 \AA, c=166.1 \AA \\
\beta=102.1^{\circ}\end{array}$ \\
\hline Resolution $(\AA)$ & $40-1.80(1.91-1.80)$ \\
\hline No. of unique reflections & $237,514(37,334)$ \\
\hline Completeness (\%) & $96.9(94.7)$ \\
\hline$R$-factor (\%) & $12.0(104.5)$ \\
\hline Multiplicity & $3.2(3.0)$ \\
\hline $\mathrm{CC} 1 / 2$ & $99.6(78.4)$ \\
\hline Mean I/ơ (I) & $5.73(1.02)$ \\
\hline \multicolumn{2}{|l|}{ Refinement } \\
\hline Resolution $(\AA)$ & $20-1.80(1.86-1.80)$ \\
\hline$R_{\text {work }} / R_{\text {free }}(\%)$ & $0.2475(0.4022) / 0.2758(0.4179)$ \\
\hline Willson B-factor $\left(\AA^{2}\right)$ & 23.62 \\
\hline No. of non-H atoms & 14,182 \\
\hline Macromolecules & 12,920 \\
\hline Ligands & 278 \\
\hline Water & 984 \\
\hline Protein residues & 1,731 \\
\hline Average $\mathrm{B}$-factor $\left(\AA^{2}\right)$ & 28.6 \\
\hline Macromolecules & 27.0 \\
\hline Ligands & 63.3 \\
\hline Water & 39.8 \\
\hline \multicolumn{2}{|l|}{ RMSDs } \\
\hline Bond length $(\AA)$ & 0.017 \\
\hline Bond angles $\left({ }^{\circ}\right)$ & 1.57 \\
\hline \multicolumn{2}{|l|}{ Ramachandran plot (\%) } \\
\hline Favored & 95.8 \\
\hline Allowed & 4.2 \\
\hline Disallowed & 0.0 \\
\hline PDB code & 7CJS \\
\hline
\end{tabular}


a

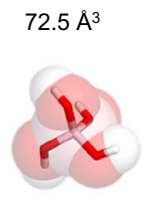

Silicic acid
$19.5 \AA^{3}$

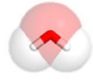

Water
$62.8 \AA^{3}$

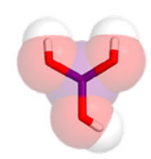

Arsenite

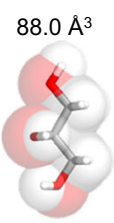

Glycerol

b

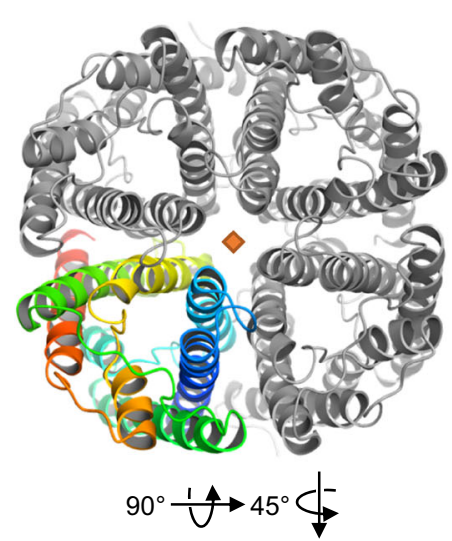

C
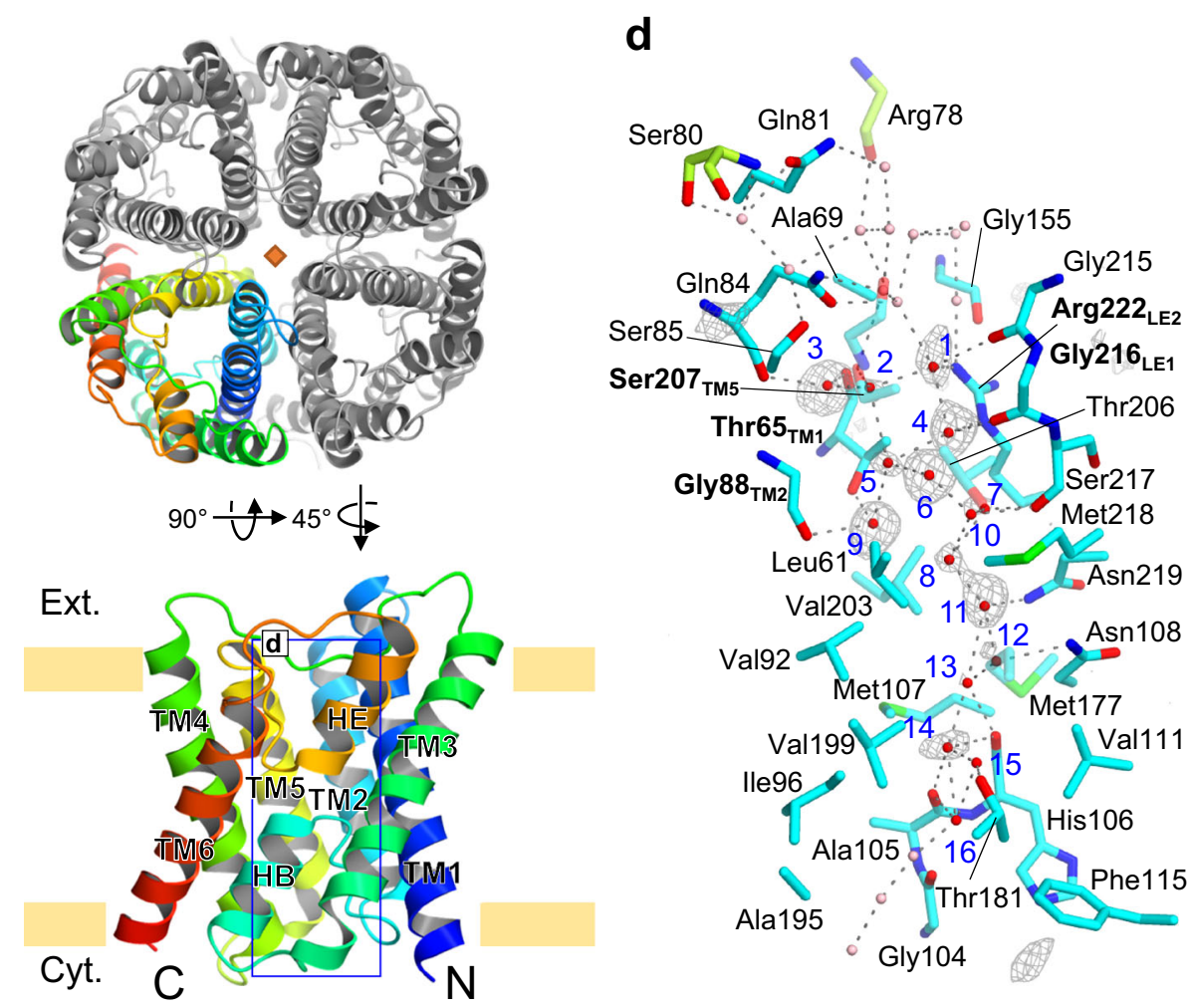

Fig. 1 Overall structure of the rice Si channel Lsi1 at a resolution of 1.8 A. a Structures and the van der Waals volumes of water, glycerol, and metalloid ( $\mathrm{Si}, \mathrm{As}$ ) transported by aquaporin. $\mathbf{b}$ The overall structure of the Lsi1 ${ }_{\text {cryst }}$ tetramer is viewed from the extracellular side. A pseudo-C4 symmetric axis is represented by a diamond shape. c Side view of the monomeric Lsi1 cryst, rainbow-colored with the $\mathrm{N}$ terminus in blue. The khaki bars indicate the membrane boundaries. d A close-up view of the channel region (chain C). Hydrogen bonding network (dot lines) shows interactions of the water molecules and the residues facing the channel's pore. Key residues comprising the ar/R SF are highlighted by bold letters. Two residues (Arg78 and Ser80) in green color are from an adjacent subunit. Water molecules positioned in the channel (red) and extra/intracellular regions (pink) are shown. Water molecules in the channel are labeled in blue, and with the omit map (gray) contoured at +3.50 .

their close approach at the extracellular side (Supplementary Fig. 5b). Also, one water molecule found in the center of bilayer regions stabilizes the arrangement of TM4 and TM5 by hydrogen bond interactions with Asn176, Thr206, and carbonyls of Val173 and Ala202 (Supplementary Fig. 5a). This water also stabilizes a conformation of Met218 in loop E that creates the hydrophobic face near the Asn-Pro-Ala (NPA) motif. Thus, Lsil $1_{\text {cryst }}$ has different TM bundle orientations in which TM1 and HE tilt largely towards the center of the channel compared to the other structurally characterized AQPs ${ }^{13,14,17-19}$ (Supplementary Fig. 4). These results reveal a structure of Lsi $1_{\text {cryst }}$ that is distinct from other AQPs, presumably associated with the high selectivity of Lsil for silicic acid since the orientation of TM helices links to the orientation of residues facing the channel.

The channel and the selectivity filter. The transport substrate specificity of AQPs is proposed to be determined by the aromatic/ Arg (ar/R) SF ${ }^{20}$, which is located below the extracellular vestibule and creates the narrowest portion of the channel. SF comprises four residues in TM2, TM5, and loop E (LE1 and LE2). SF of animal, bacterial, and archaeal AQPs are classified into two types, the water-specific AQP type ${ }^{13}\left(\mathrm{Phe}_{\mathrm{TM} 2} / \mathrm{His}_{\mathrm{TM} 5} / \mathrm{Cys} \mathrm{LE}_{\mathrm{LE}} / \mathrm{Arg}_{\mathrm{LE} 2}\right)$ or the glycerol-permeable aquaglyceroporin type ${ }^{14}\left(\operatorname{Trp}_{\mathrm{TM} 2} / \mathrm{Gly}_{\mathrm{TM} 5} /\right.$ $\left.\mathrm{Phe}_{\mathrm{LE} 1} / \mathrm{Arg}_{\mathrm{LE} 2}\right)$, both of which contain two bulky amino acid residues in the TM2/TM5/LE1 portion (Fig. 3). However, SF of Lsi1 contains three small residues $\left(\mathrm{Gly}_{\mathrm{TM} 2} / \mathrm{Ser}_{\mathrm{TM}} / \mathrm{Gly}_{\mathrm{LE} 1} /\right.$ $\left.\operatorname{Arg}_{\mathrm{LE} 2}\right)^{10}$. Based on the crystal structure, the conduction pore is $\sim 30 \AA$ long and constricts to $\sim 3.5 \AA$ at its narrowest region (Fig. 2c, d). Each monomer has 33 water molecules in the extracellular vestibule, 25 water molecules in the intracellular vestibule, and 16 water molecules in the channel (Fig. 1d and Supplementary Fig. 6c). The extracellular vestibule is hydrophilic and binds more water molecules, whereas the intracellular side is hydrophobic and bounds fewer water molecules. The contrasting environments may affect the energetic barrier to pass SF in removing hydrated water molecules or promoting hydration and releasing the transported silicic acid. This hypothesis is supported by molecular dynamics (MD) simulations of silicic acid desolvation as shown in Supplementary Fig. 6a, b). The 16 water molecules (Wat1-Wat16) in the channel were independently 

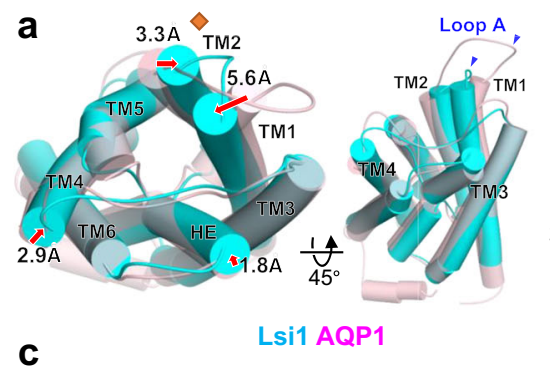

C

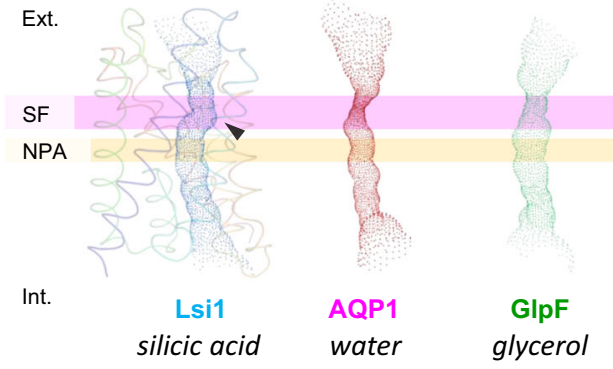

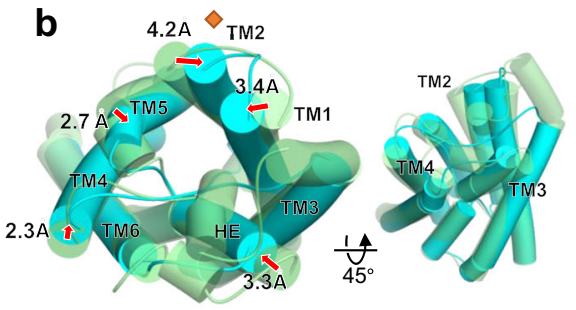

d

Lsi1 GlpF

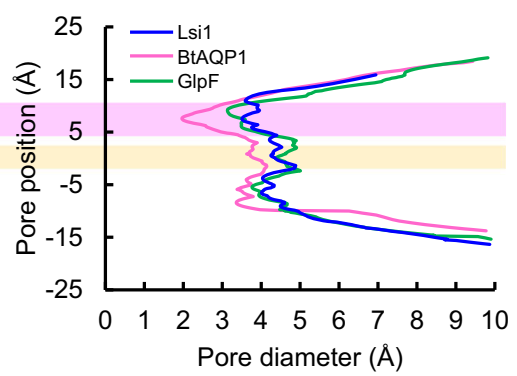

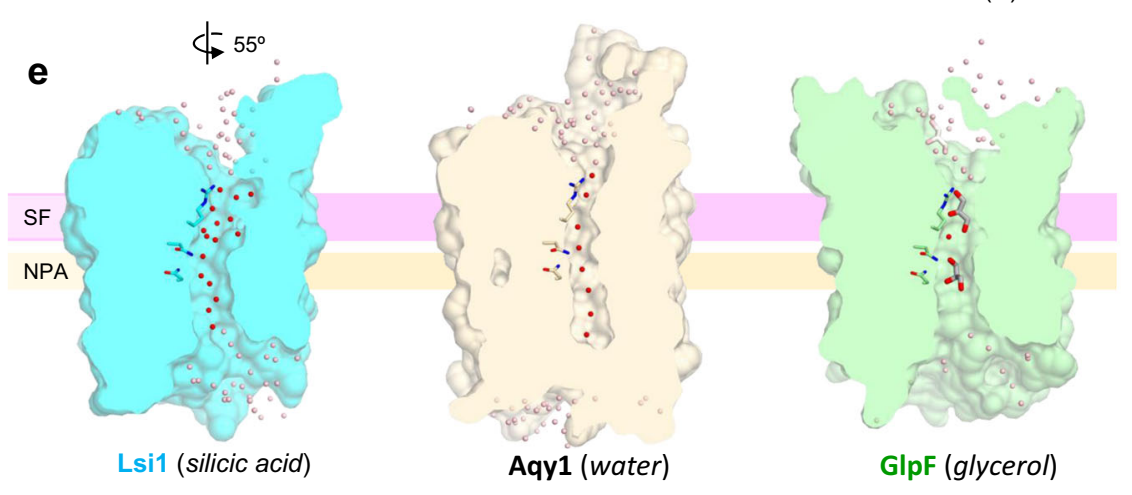

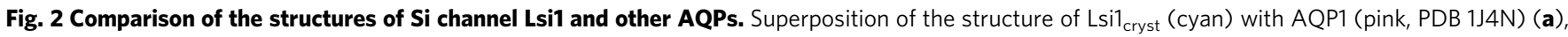
and GlpF (light green, PDB 1FX8) (b). Top view from the extracellular side (left) and a rotated view by $45^{\circ}$ against the membrane normal (right). Arrowheads indicate loop $A$ and red arrows represent the shift of TM helices (in $\AA$ ). A diamond shape represents a pseudo-C4 symmetric axis. Channel profile (c) and diameters (d) along the pore for Lsi1 cryst, AQP1, and GlpF, calculated using the program HOLE2, are shown. The regions for SF and NPA motifs are colored in plum and khaki, respectively. An arrowhead indicates the distortion in the channel. e Cross-section of the channel of Lsi1 cryst $_{\text {, Aqy } 1}$ (wheat, PDB 3ZOJ), and GlpF with a 55 rotation relative to (c). Water molecules in the channel (red), extra/intracellular regions (pink), and glycerol molecules are shown. In e, N-terminal and C-terminals of Aqy1 were omitted for clarity. The color coding shown here is used for all Figures unless otherwise noted.

identified in two non-crystallographic symmetry-related Lsi $1_{\text {cryst }}$ tetramers, indicating that they are intrinsically associated with the monomer. Wat7 and Wat8 had relatively higher temperature factors, and their positions were too closely spaced $(2.3 \AA)$ to be simultaneously occupied, suggesting that a single water molecule occupies in rapid equilibrium between adjacent sites. Similarly, several pairs of two adjacent water molecules, Wat6-Wat10, Wat11-Wat12, Wat12-Wat13, Wat13-Wat14, and Wat14-Wat15, may be occupied by a single water molecule. By contrast, Wat3 and Wat9 have typical hydrogen bonds interactions shorter than $2.8 \AA$ with the low-temperature factors, suggesting their stable binding to the channel.

A striking feature of the channel of Lsi $1_{\text {cryst }}$ is that a large number of water molecules at the extracellular half of the pore are not in a single file, which is brought by the hydrophilic SF in the broad channel (Figs. 1d and 2e). In chain A, three water molecules in SF (Wat4, Wat5, and Wat6) and two water molecules beneath it (Wat7 and Wat8) are separated by more than $3.4 \AA$ along the channel, which no longer contributes to the hydrogen bond interactions. In addition, since Wat 9 donates hydrogen bonds to the carbonyls, it cannot transfer protons to water molecules Wat5 and Wat8. It has been proposed that strongly correlated movements of the well-oriented single-file water in orthodox AQP family proteins prevent proton transfer via the grotthuss mechanism ${ }^{21}$. Lsi1 must have a different mechanism that prevents the fast proton transport since the water molecules in SF of Lsil are not single-file. The breakage of the connectivity between the SF waters and nearby water molecules may prevent proton translocation. The water molecules at the NPA motifs and the intracellular half, by contrast, are in a single file like the other AQP structures (Figs. 1d and 2e). The NPA motifs stabilize each other creating a positive electrostatic potential that functions as a barrier against proton transport across the membrane ${ }^{21-23}$. Therefore, the NPA motifs and water molecules nearby function to prevent protons in the rice $\mathrm{Si}$ channel like the other AQPs ${ }^{21-23}$.

Unlike AQP1 and GlpF, the channel of Lsi $1_{\text {cryst }}$ has a broader pore diameter at the constricted region with a slight distortion (An arrowhead in Fig. 2c). The shift of HE provides this channel's distortion, which could prevent the transport of substrates larger than silicic acid, such as silicic acid oligomer, as they are unlikely to rotate freely in this distortion. The narrowest region of the Lsil structure is located at SF, as observed in other AQPs. Two carbonyls of Gly215 and Gly216 ${ }_{\mathrm{LE} 1}$ create the constrictions that 
a

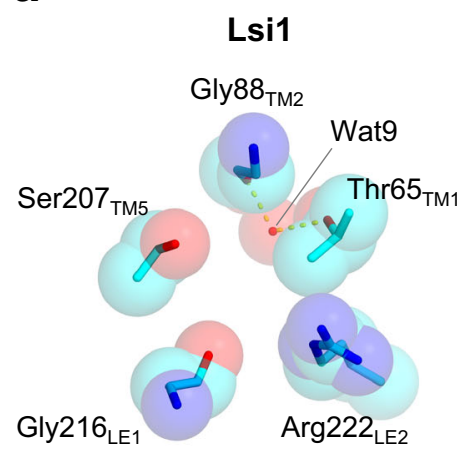

d

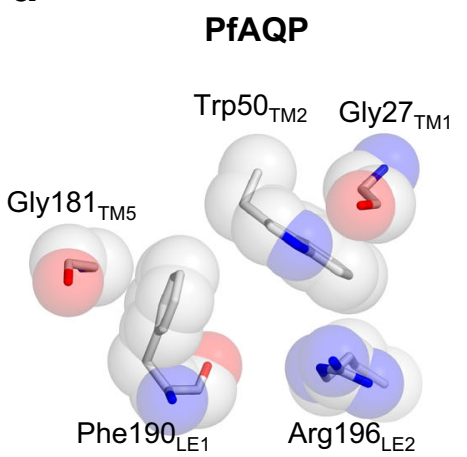

g

\section{SoPIP2;1}

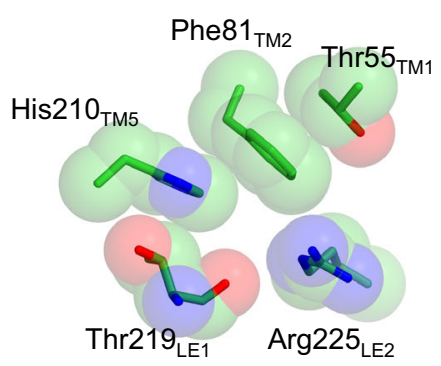

b

BtAQP1

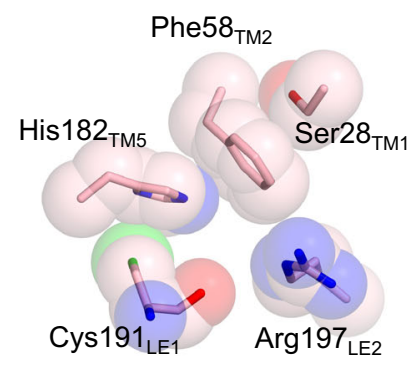

C

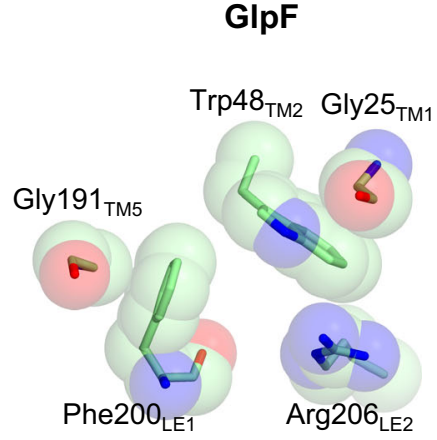

e

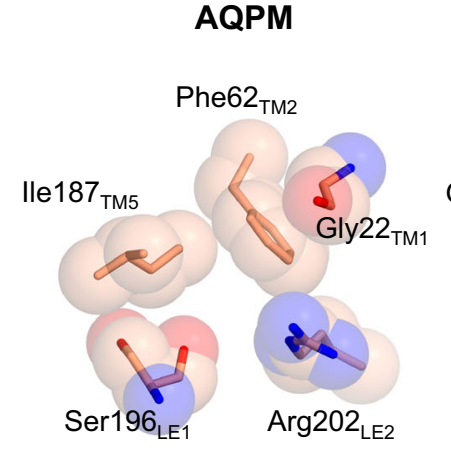

f

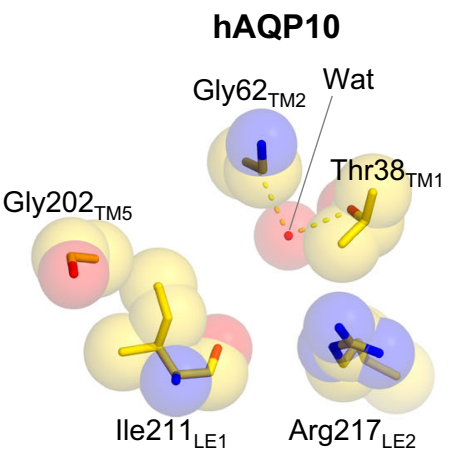

h

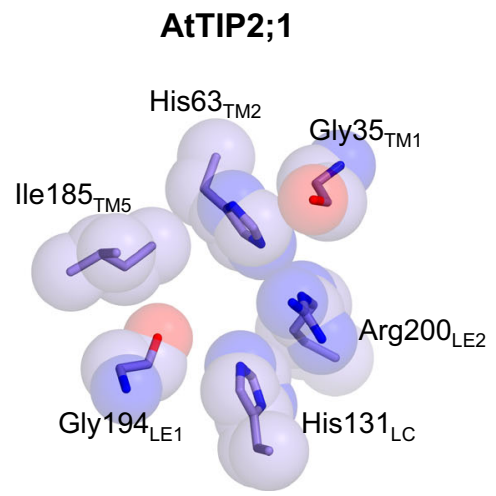

Fig. 3 Comparison of SFs of Lsi1 and other AQPs. SF of Lsi1 $1_{\text {cryst }}$ (a, cyan, PDB 7CJS), BtAQP1 (b, pink, PDB 1J4N), GlpF (c, light green, PDB 1FX8), PfAQP (d, white, PDB 3C02), AQPM (e, khaki, PDB 2F2B), hAQP10 (f, yellow, PDB 6F7H), SoPIP2;1 (g, green, PDB 1Z98), and AtTIP2;1 (h, light purple PDB 5I32). The view directions are the same as Fig. $2 \mathrm{a}$.

point towards the channel. SF of AQP1 is narrower and hydrophilic (Phe58 $\left.{ }_{\mathrm{TM} 2} / \mathrm{His} 182_{\mathrm{TM} 5} / \mathrm{Cys} 191_{\mathrm{LE} 1} / \operatorname{Arg} 197_{\mathrm{LE} 2}\right)$, and that of GlpF is wider and amphipathic (Trp48 TM2 $_{2} / \mathrm{Gly}_{191} 1_{\mathrm{TM} 5} /$ Phe $200_{\text {LE1 }} /$ Arg206 $_{\text {LE2 }}$ ), whereas that of Lsi1 $1_{\text {cryst }}$ is the widest and hydrophilic (Thr65 $5_{\mathrm{TM} 1} / \mathrm{Gly} 88_{\mathrm{TM} 2} / \mathrm{Ser} 207_{\mathrm{TM} 5} / \mathrm{Gly}_{216_{\mathrm{LE} 1} /}$ Arg222 ${ }_{\text {LE2 }}$ ) (Fig. 3). The most striking feature of the Lsi1 $1_{\text {cryst }}$ 's SF arises from an additional "fifth residue" Thr65 in TM1 $\left(\mathrm{Thr} 65_{\mathrm{TM} 1}\right)$ and a water molecule hydrogen-bonded to Thr65 $5_{\mathrm{TM} 1}$ (Wat9) (Fig. 3a). The significance of Thr65 ${ }_{\mathrm{TM} 1}$ in SF has not been recognized since, in canonical AQPs, a bulky hydrophobic residue in TM2 (Phe58 $8_{\mathrm{TM} 2}$ in AQP1 and Trp48 $8_{\mathrm{TM} 2}$ in GlpF, respectively) shields the residue equivalent to Thr65 $5_{\mathrm{TM} 1}$. In Lsi $1_{\text {cryst }}$, however, Gly88 ${ }_{\mathrm{TM} 2}$ exposes Thr65 $5_{\mathrm{TM} 1}$ to the channel, thereby making SF wide and hydrophilic. Thr65 $5_{\mathrm{TM} 1}$ donates a strong hydrogen bond to the carbonyl of Leu61 and acts as a suitable hydrogen acceptor from a nearby water molecule Wat9 (Fig. 4a). Wat9 donates hydrogen bonds to both Thr65 $5_{\mathrm{TM} 1}$ and carbonyl of Gly88 $8_{\mathrm{TM} 2}$, thereby pointing its oxygen atom towards the channel (Fig. 4). Similarly, Wat3 can donate hydrogen bonds to both carbonyls of Gln84 and Thr65 $5_{\mathrm{TM} 1}$, thereby directing its oxygen atom towards the channel as well (Fig. 4). Wat3 and Wat9 are located on one side of the channel and are separated by $6 \AA$, facing oppositely to carbonyls of Gly215 and Ser217 (Figs. 1d and 4). Therefore, unlike other AQPs, the channel of Lsil $1_{\text {cryst }}$ has two polar faces. The additional polar face's water molecules likely act as hydrogen bond acceptors during the substrate transport in a similar fashion to the consecutive carbonyls protruding the channel. This notion is supported by the lower temperature factors of Wat 3 and Wat9. 
a
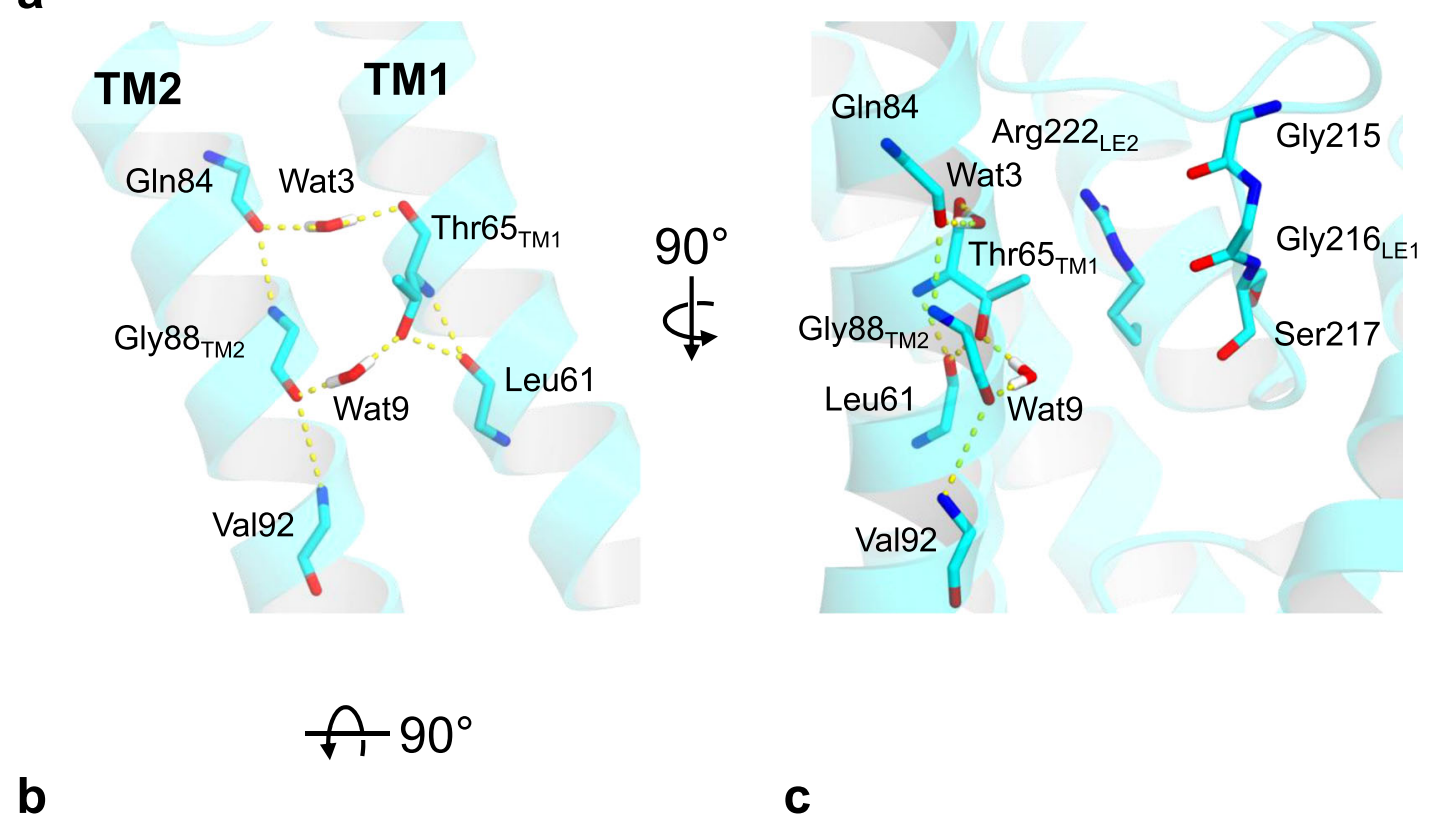

C
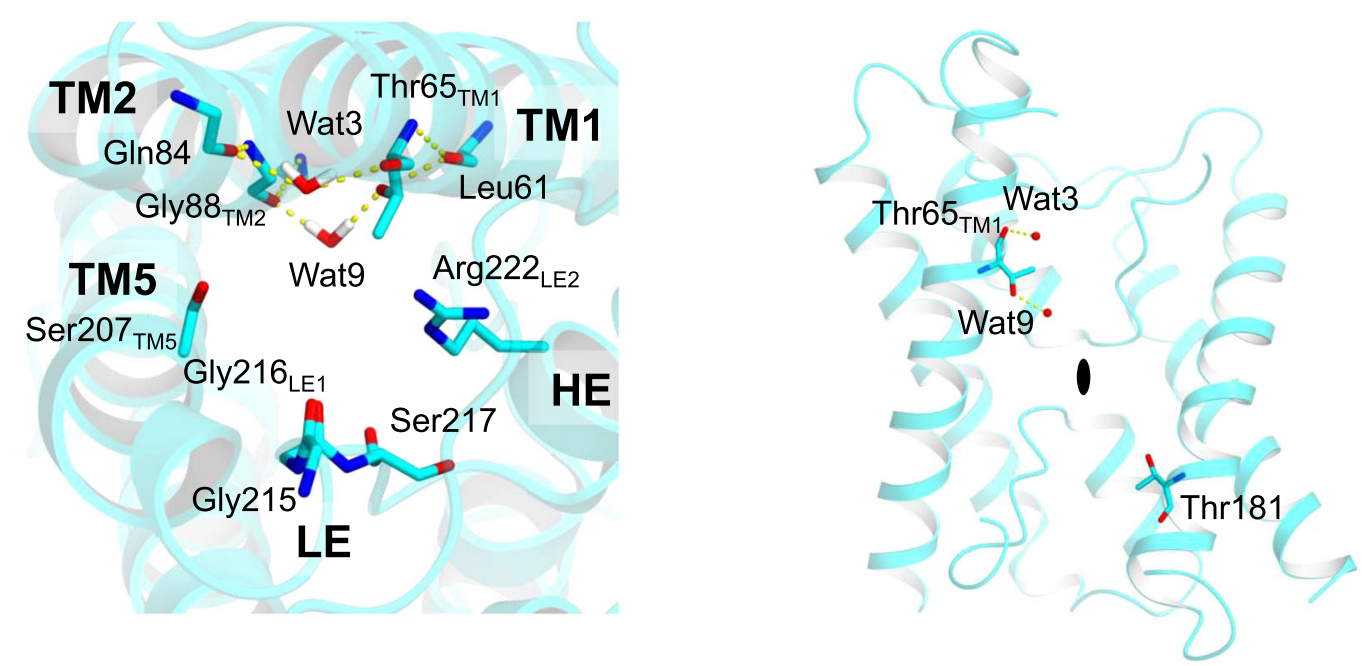

Fig. 4 Unique water molecules and two Thr residues in the Lsi1 structure. Hydrogen bonding interactions between the two water molecules comprising SF (Wat3 and Wat9) and TMs (TM1 and TM2) are shown with view directions facing the channel (a) or from the extracellular side (b). c A panel is showing the positions of Thr65 and Thr181. Hydrogen bond interactions that make the oxygen atoms of the waters pointing towards the channel are shown in yellow dashes.

Interestingly, the channel of Lsi1 $1_{\text {cryst }}$ has Thr181 in a pseudoc2-symmetrically related position ( $\mathrm{N}$ - and $\mathrm{C}$ - terminal three $\mathrm{TM}$ repeats) corresponding to the Thr65 $5_{\mathrm{TM} 1}$. Thr181 makes the channel hydrophilic likewise by having hydrogen bonds with the carbonyl of Met177 and Wat15 (Fig. 1d). These two Thr residues (Thr65 ${ }_{\mathrm{TM} 1}$ and Thr181) highlight the unique characteristic of the Si-channel Lsi1.

To examine the role of the Thr65 $5_{\mathrm{TM} 1}$ in transport substrate specificity, we generated ten variants of Lsil. We investigated their transport activity for germanic acid $(\mathrm{Ge})$ as a $\mathrm{Si}$ analog and arsenite (As) in Xenopus oocytes. The transport activities for both Ge and As were unaffected by the substitutions of Thr65 $5_{\mathrm{TM} 1}$ to Ala, Gly, and Ser (Fig. 5a, b), although expression of the Ala mutant was lower than wild type or the other two mutants in Xenopus oocytes (Supplementary Fig. 7a, b). One possible interpretation is that water molecules occupying free space created by the substitutions can compensate for a polar environment made by hydroxyl of Thr65 $5_{\mathrm{TM} 1}$ and Wat9. In contrast, the activity was substantially decreased or lost by the other substitutions (Fig. 5a, b). By T65V $\mathrm{V}_{\mathrm{TM} 1}$ substitution, which increases the hydrophobicity of SF but keeps its size unchanged, transport activities for $\mathrm{Ge}$ and As were decreased. On the other hand, the $\mathrm{T} 65 \mathrm{I}_{\mathrm{TM} 1}$ substitution that mimics the size of $\mathrm{Thr}$ residue plus Wat9 severely decreased the activity for Ge, whereas the activity for As was similar to the $\mathrm{T} 65 \mathrm{~V}_{\mathrm{TM} 1}$ mutant. These results suggested that Thr65 $5_{\mathrm{TM} 1}$, together with Wat9, constitute SF and play a key role in the specificity of transport substrate.

We also examined the role of the Thr181 in the substrate specificity by generating five site-directed mutants (T181S, T181V, T181N, T181Q, and T181D) and determined transport activity of Ge and As likewise in Sf9 cells. While the substitutions of Thr181 to Ser, Val, Asn, and Asp unaffected or slightly decreased the Ge transport activity, they slightly increased activity for As transport (Fig. 5c, d and Supplementary Fig. 7c, d). T181Q substitution, which narrows the size of the channel, completely abolished the Ge transport activity, whereas the As transport 
a

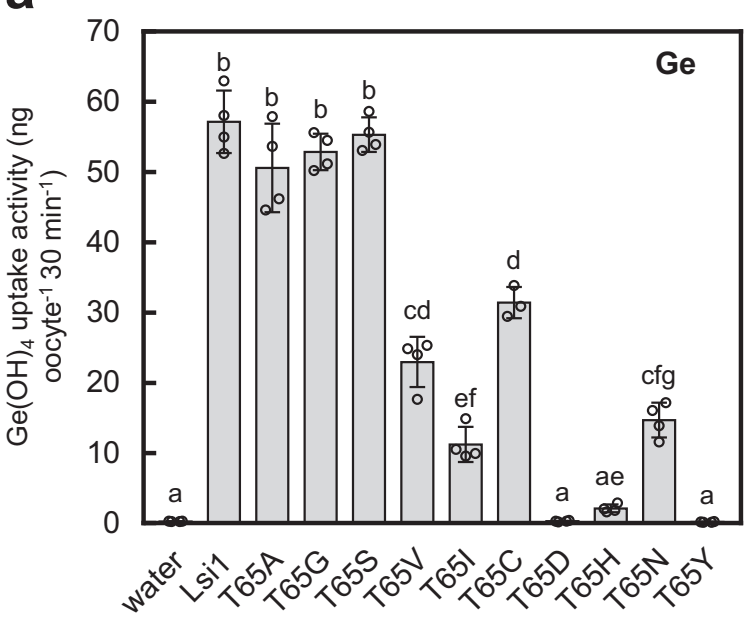

C

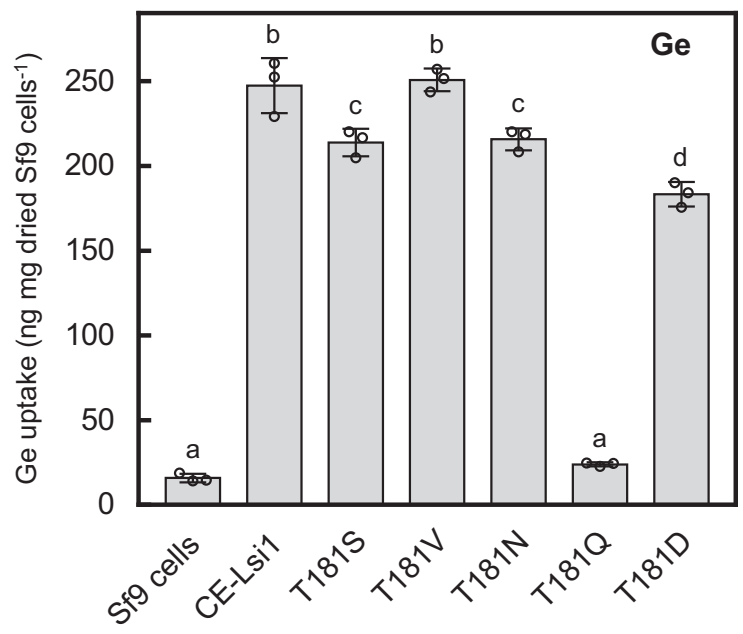

e

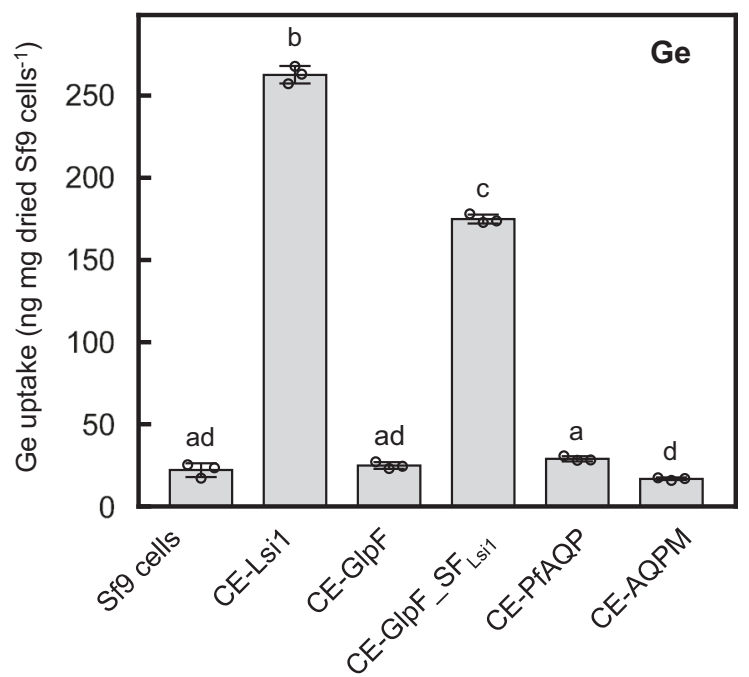

b

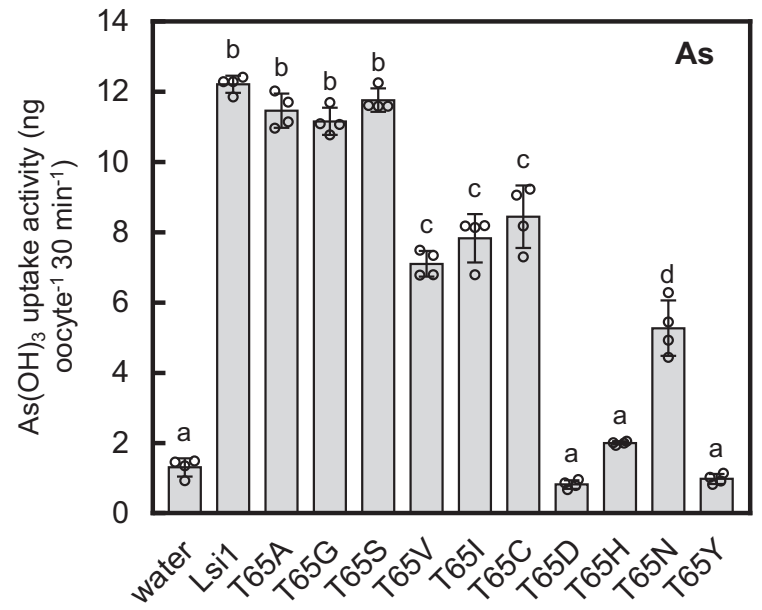

d

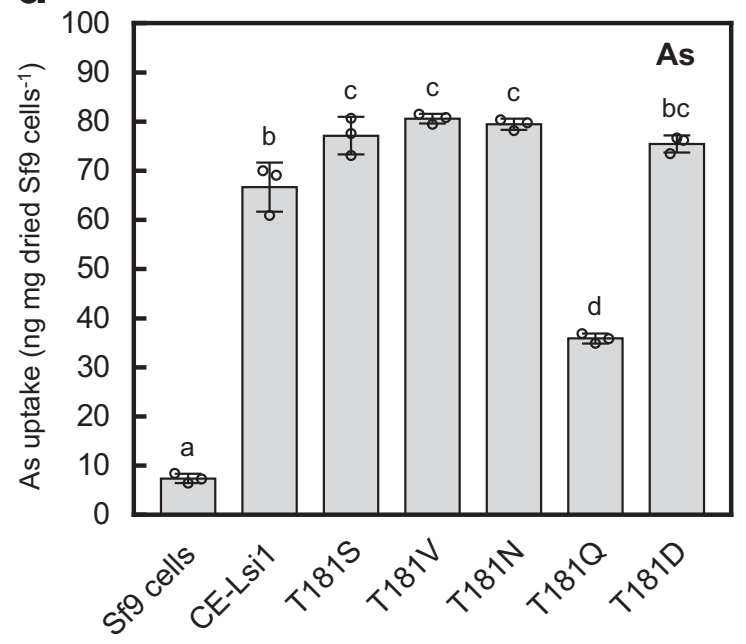

f

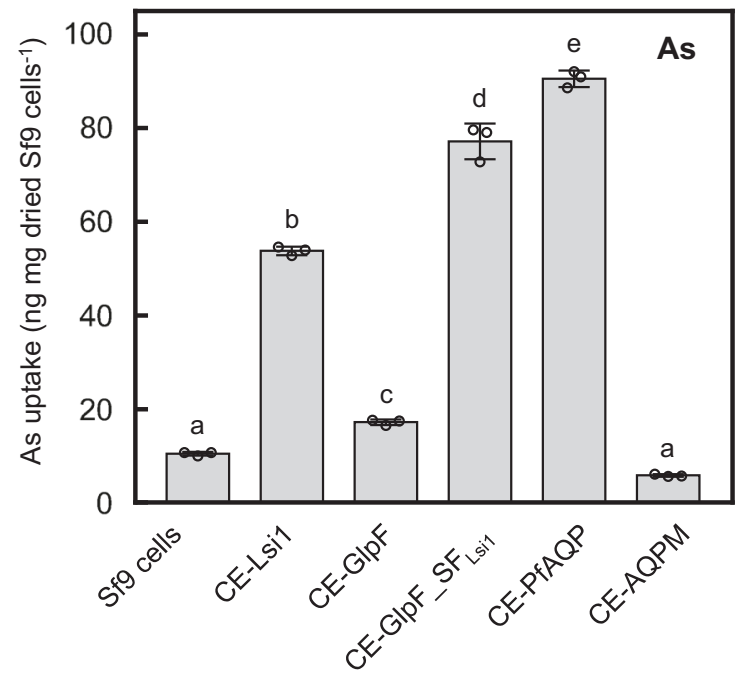

Fig. 5 Effect of Lsi1 mutation on the transport activity for Ge and As. Transport activity of germanic acid (Ge) (a, Si analog) and arsenite (As) (b) in Xenopus oocytes. The oocytes were injected with water (as a control) or cRNA of Lsi1 WT or Thr65TM1 mutants. Oocytes were exposed to a solution containing Ge or As for 30 min. Transport activity of Ge (c, e) and As (d, f) in Sf9 cells. C-terminally EGFP-tagged Lsi1 or Thr181 mutants (c, d) or other aquaglyceroporins $(\mathbf{e}, \mathbf{f})$ were expressed in the cells. The cells were exposed to a solution containing $\mathrm{Ge}$ or As for 5 min. In $\mathbf{a}-\mathbf{f}$, different letters above the columns indicate statistically significant differences at $P<0.01$ by Tukey-Kramer's test, and the test was two-sided. Values are means \pm s.d., $n=3$ for $\mathrm{T} 65 \mathrm{C}$ in (a) and $n=4$ for the others, $n$ is independent experiments. 

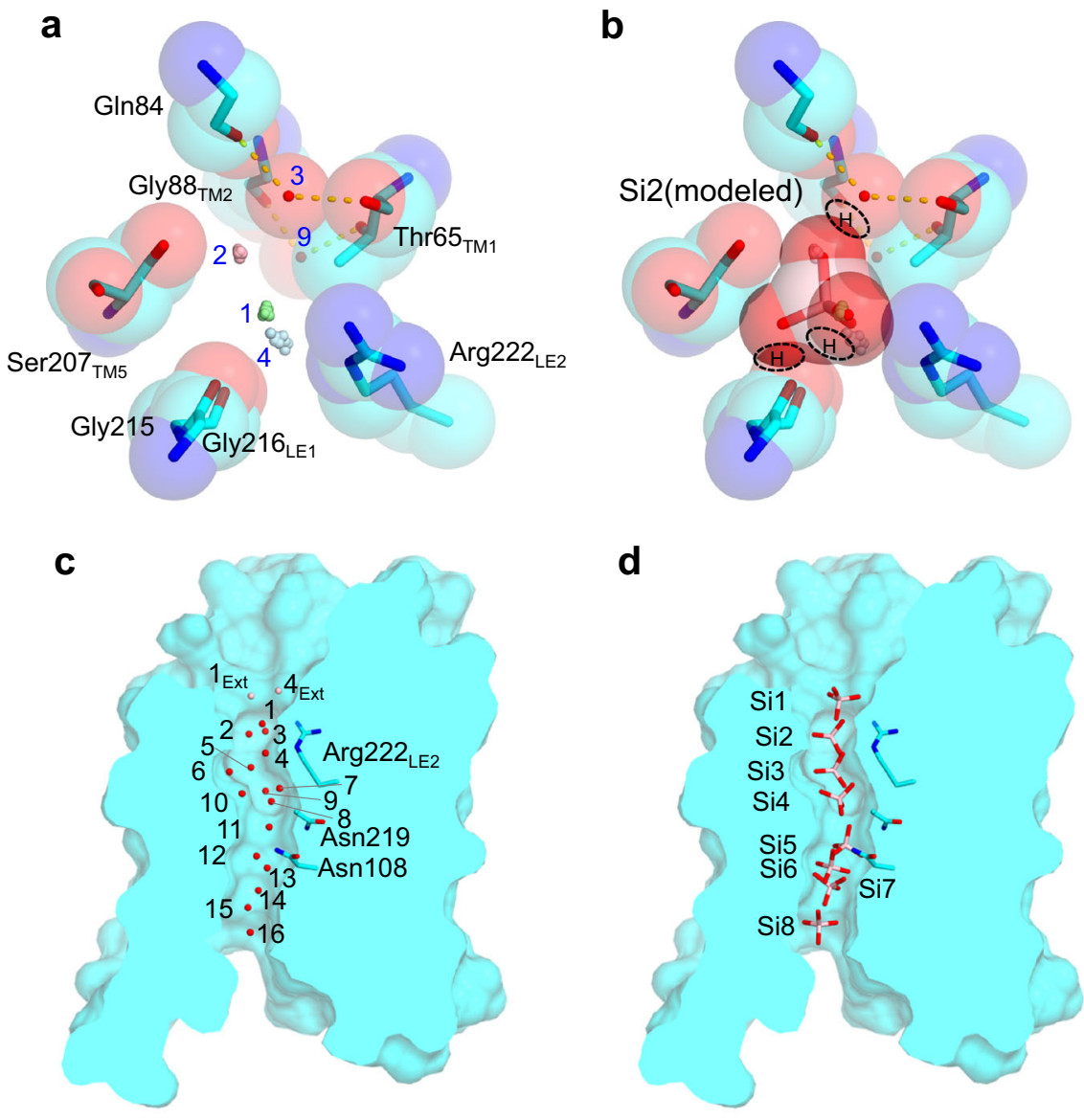

\begin{tabular}{|c|c|c|c|c|}
\hline & OH_1 & OH_2 & OH_3 & $\mathrm{OH}_{-} 4$ \\
\hline $\mathrm{Si}(\mathrm{OH})_{4} \_1$ & Ext4 & none & Ext1 & Wat1 \\
\hline $\mathrm{Si}(\mathrm{OH})_{4} \_2$ & Wat1 & none & Wat2 & Wat4 \\
\hline $\mathrm{Si}(\mathrm{OH})_{4}{ }_{3} 3$ & Wat4 & Wat6 & Wat5 & Wat7/Wat8 \\
\hline $\mathrm{Si}(\mathrm{OH})_{4} \_4$ & Wat7 & Wat10 & Wat8 & none \\
\hline $\mathrm{Si}(\mathrm{OH})_{4} \_5$ & Wat11 & none & none & Wat12 \\
\hline $\mathrm{Si}(\mathrm{OH})_{4} \_6$ & Wat12 & none & Wat13 & none \\
\hline $\mathrm{Si}(\mathrm{OH})_{4}{ }^{7}$ & Wat13 & Wat15 & Wat14 & Wat15 \\
\hline $\mathrm{Si}(\mathrm{OH})_{4} \_8$ & none & Wat15 & none & Wat16 \\
\hline
\end{tabular}

Fig. 6 Structural determinant for the silicic acid permeability and modeled eight silicic acid positions in the Lsi1 channel. SF of Lsi1 $1_{\text {cryst }}$ (a), and a possible conformation of silicic acid during the passage through SF (b). Water molecules in the channel (Wat1, Wat2, Wat3, Wat4, and Wat9) are shown as spheres and numbered in blue. Among them, three (Wat1, Wat2, and Wat4) are superposition from the eight Lsi1 $1_{\text {cryst }}$ protomers of the two tetramers. Putative hydrogens of hydroxyl groups of the silicic acid in the position Si2 are encircled by dashed lines. The view direction is the same as Fig. 2a. Water molecules used for the assignment of possible silicic acid positions (c) and the assigned eight silicic acids (Si1-Si8) in the channel (d) are shown in a crosssection view. Water molecules in the channel region (red spheres) and the extracellular region (pink spheres) are labeled in numbers. Arg222 LE2 $_{2}$ in the SF region and two Asn residues in the NPA motifs are shown as the stick. e Water molecules employed for the assignment of four hydroxyl groups in silicic acid are listed. Ext in $\mathbf{c}$ and $\mathbf{e}$ indicates water molecules at the extracellular side.

activity remained about $35 \%$ of native Lsi1 (Fig. $5 \mathrm{~d}$ ). It should be noted that the effect of Thr181 mutations is moderate compared to Thr65 $5_{\mathrm{TM} 1}$ and its function is still not clear. Nevertheless, the results suggest that the specificity of transport substrate can be modified by manipulating the two Thr residues (Thr65 $5_{\mathrm{TM} 1}$ and Thr181) identified in the present study.

Transport specificity of Lsil for silicic acid. What is the structural basis of Lsil yielding the high selectivity for silicic acid rather than smaller glycerol? Our structural analysis showed that the oppositely located two polar faces found in the channel might be responsible for this difference in the transport substrate specificity (Fig. 3a). Since silicic acid is a hydrophilic tetrahedron, the two polar faces can provide an energetically preferable environment by surrounding it. In contrast, linear carbohydrates are amphipathic, and the two polar faces in Lsi $1_{\text {cryst }}$ may impair conductivity for glycerol. Indeed, SF of GlpF has an amphipathic property that fits well for glycerol with hydrophobic interactions by $\operatorname{Trp} 48_{\mathrm{TM} 2}$ and Phe200 ${ }_{\mathrm{LE} 1}$ and hydrogen bonds formed by Arg206 2 LE2 and carbonyl of Phe200 LE1 with the glycerol's hydroxyl 

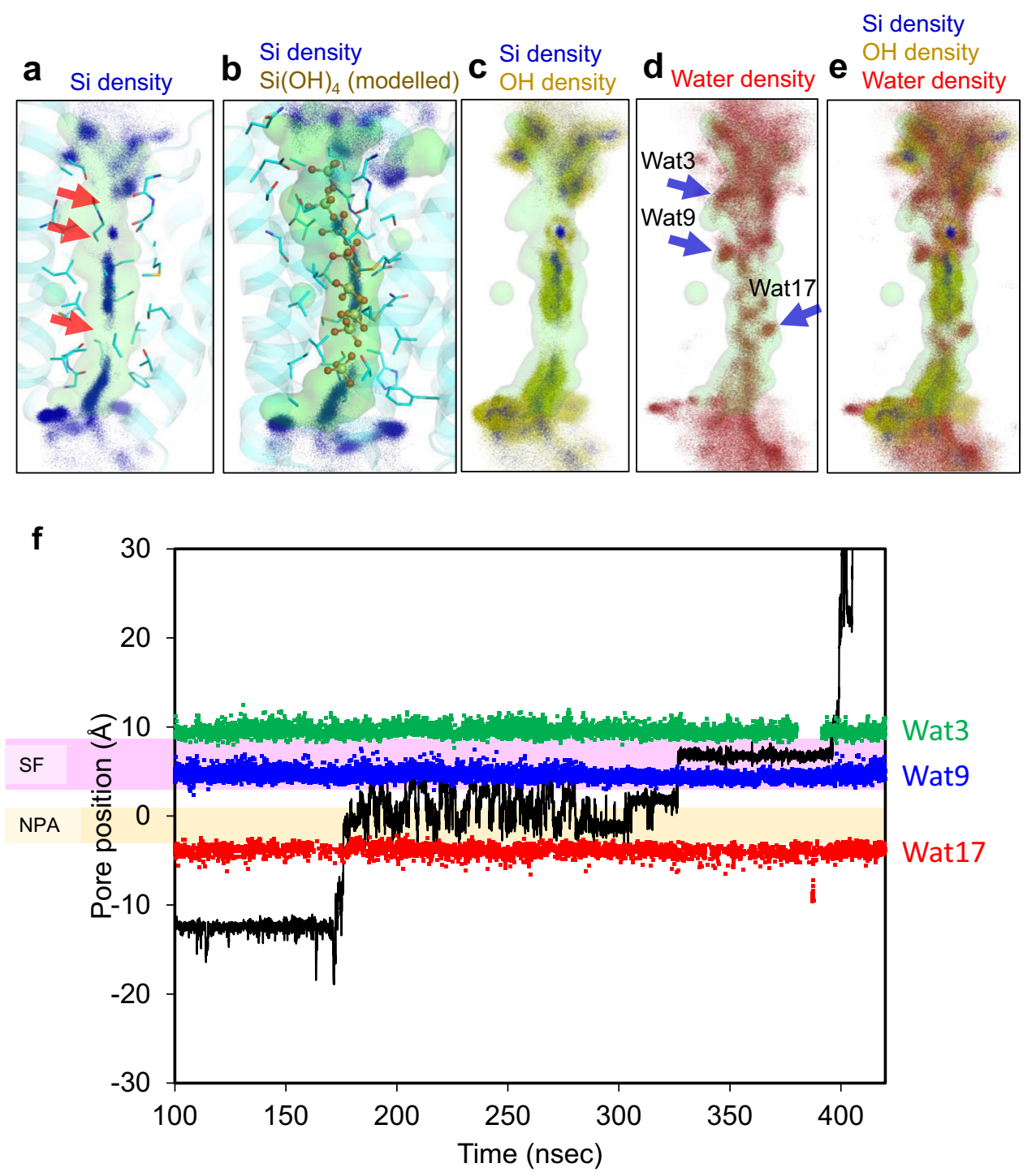

Fig. 7 MD simulation of Lsi1 with silicic acid. Distribution of $\mathrm{Si}$ atoms of silicic acid (a) and its overlay with modeled $\mathrm{Si}(\mathrm{OH})_{4} \mathrm{molecules}(\mathrm{Si1}-\mathrm{Si} 8)(\mathbf{b})$, and distribution of $\mathrm{Si}$ atoms as well as oxygen atoms of silicic acid (c), or oxygen atoms of water (d) in the 0-300 ns MD simulation. In a, structural bottlenecks found in the channel are indicated by red arrows. In d, Wat3, Wat9, and Wat17 locations are indicated by blue arrows. Distributions of (c) and (d) are merged in (e). $\mathbf{f}$ The MD trajectory of the silicic acid permeation during the 100-420 ns. Positions of silicic acid (black), Wat3 (green), Wat9 (blue), and Wat17 (red) in the channel are plotted. Enlarged views of (f) and the distributions of $\mathrm{Si}(\mathrm{OH})_{4}$ molecules at the beginning and end of the simulation are provided in Supplementary Fig. 11.

groups (Fig. 3c). To test this possibility, we focused on gain-offunction mutants of the structurally characterized aquaglyceroporin $\mathrm{GlpF}^{14}$ in an attempt to mimic the Si-permeability of Lsi1. Wild-type GlpF, PfAQP24, and AQPM ${ }^{25}$ did not show transport activity for Ge in Sf9 cells or oocytes (Fig. 5e and Supplementary Figs. 7c, d, 8e). However, with the substitutions of their SF residues into Lsil type, GlpF variant $\mathrm{GlpF}_{-} \mathrm{SF}_{\mathrm{Lsi1}}$ $\left(\mathrm{G}_{25 \mathrm{~T}} \mathrm{~T}_{\mathrm{TM}}, \mathrm{W} 48 \mathrm{G}_{\mathrm{TM} 2}, \mathrm{G} 191 \mathrm{~S}_{\mathrm{TM} 5}\right.$, and $\left.\mathrm{F} 200 \mathrm{G}_{\mathrm{LE1}}\right)$ showed a higher Ge transport activity (Fig. 5e and Supplementary Fig. 8e). Interestingly, $\mathrm{GlpF}_{-} \mathrm{SF}_{\mathrm{Lsi}}$ also gained the As transport activity but showed the decreased glycerol transport activity (Fig. $5 f$ and Supplementary Fig. 8c, f).

Deshmukh et al. reported that number of residues connecting two NPA motifs is essential for the Si permeability ${ }^{26}$. Since the spacing is 108 residues in Lsil and 132 residues in the Sipermeable GlpF variant $\mathrm{GlpF}_{-} \mathrm{SF}_{\mathrm{Lsil}}$, the spacing itself is unlikely to be critical for the Si specificity. This is supported by a recent study on Lsil in tomatoes that possesses 109 residues in the spacing but shows transport activity for $\mathrm{Si}^{27}$. Instead, loop C extensively stabilizes the consecutive carbonyls (Gly215-Ser217) and SF of Lsi $1_{\text {cryst }}$ via hydrogen bond interactions (Supplementary Fig. 5b). Taken together, the two polar faces of the channel provided by the unique SF have an essential role in the $\mathrm{Si}$ specificity in Lsil.

The silicic acid transport mechanism. We failed to detect any Sior Ge- derived anomalous signals from the Lsi $1_{\text {cryst }}$ crystals soaked in a buffer at saturated concentrations. However, we tentatively predicted eight positions of silicic acid (Sil through Si8) that possibly occupy the channel in Si transport, based on the positions of waters identified in the channel (Watl through Wat16) (Fig. 6a). We postulated that hydroxyl groups of the transported silicic acid should favor the hydrophilic environment that waters bound. We first investigated the modeled silicic acid locations using quantum mechanical/molecular mechanical (QM/MM) 


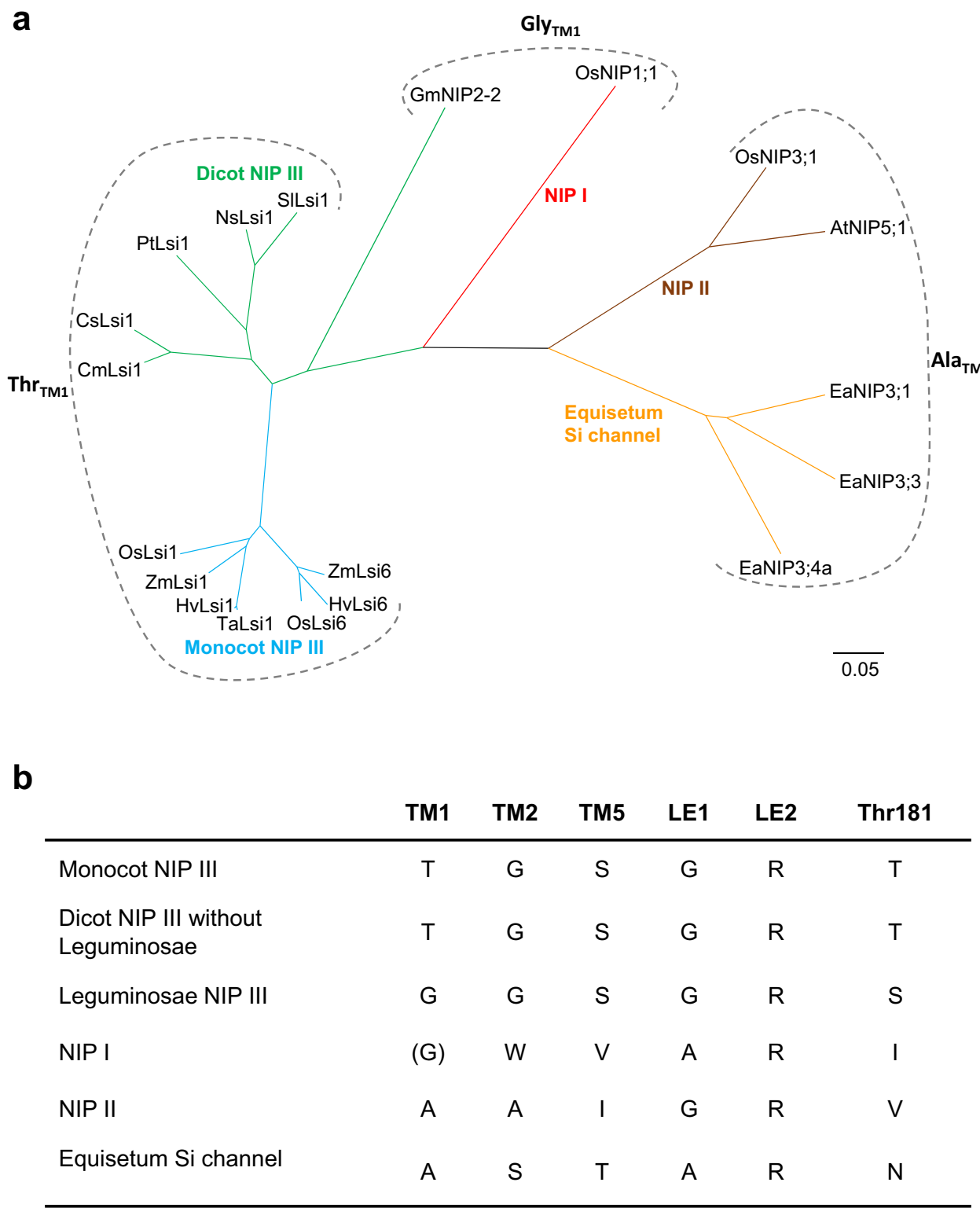

Fig. 8 Analysis of selectivity filters in NIP subfamilies. Phylogenetic tree of plant NIP subfamilies (a), and summary of the residues comprising the selectivity filer and Thr181 (b). The phylogenetic tree was constructed using the neighbor-joining algorithm by the software MEGAX ${ }^{51}$ after the sequence alignment using Clustal Omega ${ }^{52}$ with 1,000 bootstrap trials. The 0.05 scale indicates substitution distance.

geometry optimization (Supplementary Fig. 9). Displacement of the $\mathrm{QM} / \mathrm{MM}$ model ranges from 0.2 to $0.9 \AA$ relative to the modeled silicic acid (Supplementary Fig. 9). The number of hydrogen-bonding interactions in the channel is mainly unchanged after the QM/MM optimization, suggesting that silicic acid can stably occupy the positions through Sil to Si8.

Intriguingly, the modeled Si2 occupies SF, without any steric hindrance, with the three hydroxyl groups overlapping with three water molecules (Wat1, Wat2, and Wat4), thereby donating hydrogen bonds to Wat3 and two carbonyls (Gly215, and Gly216 LE1) (Fig. 6a, b). Si2 thus likely represents a transient conformation during the passage through SF. Two hydroxyl groups go through a crevice formed by Arg222 $2_{\mathrm{LE} 2}$ and the two carbonyls (Gly215 and Gly216 ${ }_{\mathrm{LE} 1}$ ), another one goes through a crevice by the carbonyls and Ser $207_{\mathrm{TM} 5}$, and the other one goes through a crevice by Ser207 TM5 and waters (Wat3 and Wat9) (Fig. 6b). In this way, all hydroxyl groups of the silicic acid can form hydrogen bonds with the carbonyls or waters positioned along the channel and with Ser207 $7_{\mathrm{TM} 5}$ and $\operatorname{Arg} 222_{\mathrm{LE} 2}$ to compensate for the energetic cost of dehydration. The other four silicic acids (Si1, Si3, Si4, and Si7) overlap three or four hydroxyl groups with the waters identified, and the other three (Si5, Si6, and Si8) also do so in two hydroxyl groups (Fig. $6 c-e)$. This assignment identifies plausible hydrogen bonding partners of the silicic acid and provides orientations of the former four positions. However, there may be other interpretations because we based our hypothesis on the structure without any substrates. The predicted silicic acids suggested that water molecules can replace one or two hydrogen-bonding interactions with the channel after the passage of SF to promote silicic acid migration.

We also performed a $450 \mathrm{~ns} \mathrm{MD}$ simulation of the Lsil structure in the presence of $\sim 1 \mathrm{M} \mathrm{Si}(\mathrm{OH})_{4}$ to investigate the transport mechanism (Fig. 7 and Supplementary Figs. 10, 11). Two silicic acids permeated the channel during the simulation. During the MD simulation, an average of $11.6 \pm 2.6$ water molecules and $0.7 \pm 0.7 \mathrm{Si}(\mathrm{OH})_{4}$ molecules occupied in the 
channel region, where we found 16 water molecules in the crystal structure (Supplementary Fig. 10f). The simulation results also suggested three structural bottlenecks where silicic acid densities were low during the simulation and silicic acid cannot move freely (Red arrows in Fig. 7a). Two bottlenecks were in SF; one was consistent with the modeled $\mathrm{Si} 2$, and the other was between the Si3 and Si4. The third one was near the Thr181 in between the $\mathrm{Si} 7$ and Si8. The simulation suggested that each bottleneck has a cavity which the oxygen atoms of silicic acid cannot occupy. Instead, water molecules, Wat3, Wat9, and Wat17 (Wat17 was not identified in the crystal structure and has hydrogen bonding interaction with Thr181 in the MD simulation, see Supplementary Fig. 10b), stably exist within the cavities (Blue arrows in Fig. 7d). When the $\mathrm{Si}(\mathrm{OH})_{4}$ moved across the Wat3 and Wat17 sites, Wat3/Wat17 were dissociated from this site. On the other hand, Wat9 was independent of the $\mathrm{Si}(\mathrm{OH})_{4}$ movement and remained bound to the site (Fig. 7f and Supplementary Figs. 10f and 11). We calculated how often these water molecules occupy the cavities throughout the MD simulation. Their occupancies were $60 \%$ for Wat $3,94 \%$ for Wat 9 , and $44 \%$ for Wat17. Their exchange rate suggested that Wat 9 bound to the site longer (the average exchange time, $\tau=1.5 \mathrm{~ns}$ ) than Wat 3 and Wat17 ( $\tau \sim 0.3 \mathrm{~ns}$ ) (Supplementary Table 2). In this case, Wat3 formed hydrogen bonding interactions with the carbonyls of Gln84 or Thr65 $5_{\mathrm{TM} 1}$ in most cases, whereas Wat9 mainly formed two hydrogen bonds with Thr65 $5_{\mathrm{TM} 1}$ and $\mathrm{Gly} 88_{\mathrm{TM} 2}$. MD snapshots also suggested that Wat 3 and Wat 9 could accept hydrogen bonds from silicic acid consistent with our proposal from the crystal structure (Supplementary Fig. 10c, e). The silicic acid permeation trajectories and the MD snapshots support that Wat3 and Wat9 remain in the vicinity of their positions during the silicic acid passage while Wat17 will be displaced (Fig. $7 \mathrm{f}$ and Supplementary Figs. 10, 11). The channel diameter calculation including Wat9 results in a narrower channel diameter (Fig. 2c and Supplementary Fig. 12). These results indicate that during silicic acid permeation, Wat 3 and Wat 9 act as part of the channel lumen, narrowing the channel and strictly selecting the orientation of silicic acid, highlighting the importance of the highresolution structure's ability to visualize most water molecules in the channel.

\section{Discussion}

Plant AQPs have five subfamilies; the plasma membrane intrinsic proteins (PIP) subfamily, the tonoplast intrinsic proteins (TIP) subfamily, the NIP subfamily, the small basic intrinsic proteins subfamily (SIP), and the X intrinsic proteins (XIP) subfamily ${ }^{28}$. In the present study, we determined the high-resolution crystal structure of the rice Si channel Lsil belonging to the unique NIP subfamily. We compared the Lsi $1_{\text {cryst }}$ structure with other known plant AQP structures, SoPIP2;1 and AtTIP2;1. The SoPIP2;1 structure in the open and closed states have been reported $^{17}$. The striking feature of the SoPIP2; 1 structure is that loop D changes its conformation to open or occlude the pore in response to phosphorylation or $\mathrm{pH}$ change. In the SoPIP2;1 structure, loop D folds below the pore and occludes it in the closed conformation, whereas loop D flips largely towards TM4 and TM5 of the adjacent protomer, thereby opening the pore in the open conformation. The overall structure of Lsil is very similar to that of SoPIP2;1 (The RMSD values are $1.1 \AA$ for the open and $1.2 \AA$ for the closed structures, respectively), except for loop D (Supplementary Fig. 13a). Loop D in the Lsil structure flips towards TM2 of the adjacent protomer (Supplementary Fig. 13b). SF of the SoPIP2;1 is narrow and hydrophilic (Phe81 $1_{\mathrm{TM} 2} / \mathrm{His}_{2} 10_{\mathrm{TM} 5} /$ Thr219 $\left.{ }_{\mathrm{LE1}} / \operatorname{Arg} 225_{\mathrm{LE} 2}\right)$ similar to other structures of the waterspecific AQPs (Fig. 3g). Thr55, which corresponds to the "fifth residue" of the Lsil structure, is covered by the Phe $81_{\mathrm{TM} 2}$ and therefore does not face the pore.

On the other hand, AtTIP2;1 is water and ammonia permeable. While the Lsil structure is similar to the AtTIP2;1 structure (the RMSD value is $1.4 \AA)^{18}$, their loops $\mathrm{A}$ and $\mathrm{C}$ are quite different. In the Lsil structure, loop A orients to the pore's distal side, and loop C is displaced up to $5 \AA$ towards the pore relative to the AtTIP2;1 structure (Supplementary Fig. 14). SF of AtTIP2;1 is narrow and hydrophilic (His63 $\mathrm{TM}_{2} / \mathrm{His} 131_{\mathrm{LC}} / \mathrm{Ile} 185_{\mathrm{TM} 5} / \mathrm{Gly} 194_{\mathrm{LE} 1} /$ Arg200 ${ }_{\mathrm{LE} 2}$ ) (Fig. $3 \mathrm{~h}$ and Supplementary Fig. 15). Gly35, which corresponds to the "fifth residue" of the Lsil structure, is covered by the bulky His63 $3_{\mathrm{TM} 2}$. SF of AtTIP2;1 is characterized by the fact that the conserved Arg200 ${ }_{\mathrm{LE} 2}$ adopts a unique position and that an additional hydrophilic residue His $131_{\mathrm{LC}}$ extended from loop C contributes to SF. Lsil has Thr157 at the corresponding position of His $131_{\text {LC }}$ in the AtTIP2;1 structure (Supplementary Fig. 15). Thr157 in Lsil has hydrogen bonding interaction with Thr223 and exposes its methyl group to the pore. Moreover, MD simulation suggested that $\mathrm{Si}(\mathrm{OH})_{4}$ remains at the position of Thr157 in the crystal structure when the Thr157 is displaced (Supplementary Fig. 15e, f). Therefore, Thr157 in Lsil is distinct from the AtTIP2;1 structure and may not directly contribute to substrate selectivity.

Among five residues defining SF of the Lsi $1_{\text {cryst }}$ structure, Thr65 $65_{\mathrm{TM} 1}$ is unique and likely plays an essential role in the specificity of the transport substrate (Fig. 3a). Among the NIP subfamily (NIP I, II, and III), NIP I subgroup has a bulky Trp TM2 $_{\text {TM }}$ in SF, whereas NIP II and III subgroups have small amino acid residues $\mathrm{Gly}_{\mathrm{TM} 2}$ or $\mathrm{Ala}_{\mathrm{TM} 2}$ or $\mathrm{Ser}_{\mathrm{TM} 2}$ (Fig. 8). Therefore, the "fifth residue" of SF corresponding to Thr65 $5_{\mathrm{TM} 1}$ in Lsil is likely to face the channel in NIP II and III subgroups but not in the NIP I subgroup. In the NIP III subgroup, the "fifth residue" is $\mathrm{Thr}_{\mathrm{TM}}$ in monocot and dicot species, except $\mathrm{Gl}_{\mathrm{TM} 1}$ in legumes, whereas it is $\mathrm{Gly}_{\mathrm{TM} 1}$ or $\mathrm{Ala}_{\mathrm{TM} 1}$ in NIP I and II subgroups (Fig. 8). Also, Thr181 is conserved in NIP III subgroup except for leguminosae (Supplementary Fig. 1). Thus Thr65 $5_{\mathrm{TM} 1}$ and Thr181 identified in the present study are unique to the NIP III subgroup. The Equisetum Si channel group, which also possesses the Si transport activity, has $\mathrm{Ala}_{\mathrm{TM} 1}$, $\mathrm{Ser}_{\mathrm{TM} 2}$, and $\mathrm{Thr}_{\mathrm{TM} 5}{ }^{29}$ (Fig. 8). Hydroxyl groups of $\mathrm{Ser}_{\mathrm{TM} 2}$ and $\mathrm{Thr}_{\mathrm{TM}}$ in the Equisetum Si channel may compensate for the substrate specificity or interactions with water molecules provided by the hydroxyl groups of Thr65 $5_{\mathrm{TM}}$ and Ser207 ${ }_{\text {TM5 }}$ in Lsil.

Human aquaglyceroporin hAQP10 is permeable to silicic $\operatorname{acid}^{30}$, and its structure in the closed state has been reported ${ }^{31}$. The RMSD value between the hAQP10 and Lsil $1_{\text {cryst }}$ structures is $1.7 \AA$, which is slightly larger than those calculated between Lsi1 and the structures SoPIP2;1 and AtTIP2;1. The relatively larger RMSD value arises from different structures in loops and different orientations of TM helices (Supplementary Fig. 16a). Nevertheless, SF of hAQP10 is very similar to that of Lsil (Fig. 3a, f), consistent with the fact that hAQP10 is permeable to $\mathrm{Si}(\mathrm{OH})_{4}$. SF of hAQP10 is wide and amphiphilic (Thr35 $5_{\mathrm{TM} 1} / \mathrm{Gly} 62_{\mathrm{TM} 2} /$ Gly202 ${ }_{\mathrm{TM} 5} / \mathrm{Ile} 211_{\mathrm{LE} 1} / \operatorname{Arg} 217_{\mathrm{LE} 2}$ ). SF of hAQP10 contains many water molecules, including two water molecules Wat 3 and Wat9, in the Lsil structure, which create the polar face (Fig. $3 \mathrm{f}$ and Supplementary Fig. 16c). There are two notable differences in SF between hAQP10 and Lsil. First, the pore diameter of the hAQP10 is wider in SF (Supplementary Fig. 16b). Second, Gly210 and Ile211 $11_{\mathrm{LE} 1}$ provide a row of carbonyls in the pore in the hAQP10 structure, but they are two Gly residues (Gly215 and Gly216 $6_{\mathrm{LE1}}$ ) in the Lsi1 structure. The side chain of Ile211 $1_{\mathrm{LE} 1}$ renders the channel hydrophobic and displaces TM5 of hAQP10 up to $5 \AA$ towards the pore's distal side relative to the Lsi1 structure. Therefore, SF's hydrophilicity and the tilting angles of TM helices are different between the structures hAQP10 and 
Lsi1 (Fig. 3f and Supplementary Fig. 4e). The bulky Ile211 $1_{\text {LE1 }}$ residue also affects the orientation of the carbonyls. The carbonyl of Gly 210 in hAQP10 rotates by $40^{\circ}$ towards Arg2 $17_{\mathrm{LE} 2}$ compared to Gly215 in the Lsil structure. This rotation weakens the hydrogen bond to waters and narrows the region of the pore through which silicic acid may pass. Given that SF of Lsil is ideal for silicic acid permeation, the selectivity for silicic acid of hAQP10 may be different from that of Lsil.

We have shown that the unique TM helix orientations and SF of Lsil are essential for silicic acid transport. Lsil has presumably acquired these features during its evolution from canonical AQPs that could not transport silicic acid. The evolution of plant AQP family proteins that permeate substrates other than water and glycerol, such as Lsil and AtTIP2;1, seems to have involved drastic modification of SF from canonical AQPs. As described, even between the evolutionarily distant species of human (hAQP10) and rice (Lsi1), a shared structure exists in which water molecules create a polar face in SF to transport silicic acid. Such a common structure has likely evolved from convergent evolution.

Lsil is also permeable to carcinogenic arsenite ${ }^{11}$, the primary form of As in the paddy field. Arsenite is also present in the form of a non-charged molecule and has a similar size as silicic acid ${ }^{11}$. Rice is a staple food for half of the world population but can accumulate high As through Lsi1 ${ }^{11}$. Since rice is the primary dietary source of As, it is crucial to reduce As in rice grain for human health. However, compared with silicic acid, usually, arsenite shows broader specificity ${ }^{12}$. For example, two T65I $\mathrm{I}_{\mathrm{TM}}$ and T181Q substitutions were identified to decrease or abolish Ge-transport activity while As-transport activity was substantially retained (Fig. 5). Also, Lsi1 with a S207I $\mathrm{I}_{\mathrm{TM} 5}$ substitution ${ }^{10}$ does not transport Ge but transports As. Silicic acid is a tetrahedral molecule that forms four hydrogen bonds, whereas arsenite is a trigonal pyramid that forms three hydrogen bonds (Fig. 1a). Since SF closely matches the transport substrates in the dehydrated form as observed in $\mathrm{Lsil}_{\text {cryst }}$ and $\mathrm{GlpF}^{14}$, or even in the KcsA potassium channel ${ }^{32}$, the larger number of possible hydrogenbonding interactions with the channel as well as the tetrahedral stereochemistry may be the reasons why selectivity for silicic acid is stricter than that for arsenite. While other factors determining transport substrate specificity remain to be investigated, the structure of Lsil obtained in this study could serve as a blueprint for rational designs of transgenic crops that specifically take up silicic acid but not arsenite through manipulating the selectivity of Lsil. Such modification will contribute to safe food production in the future.

\section{Methods}

Protein expression and purification of Lsi1. The Lsi1 gene from rice (Oryza sativa cv. Nipponbare) was cloned into the pFastBacl vector for baculovirus expression in Sf9 insect cells using standard methods. A TEV protease cleavage site and the octa-His affinity tag were introduced between the $\mathrm{C}$ terminus of Lsil and EGFP. The functionally active construct of Lsil was discovered by examining Nand C-terminal deletion constructs, several point mutations, as well as additional $\mathrm{Si}$ permeable AQPs from other organisms. All these constructs were created by using the QuikChange II site-directed mutagenesis method (Stratagene) with primers (Supplementary Table 3 ) and screened by FSEC ${ }^{15}$. Removing 44 residues from the $\mathrm{N}$-terminus, 24 residues from the C-terminus, with seven point mutations (K50R, C66A, T93V, C139A, K232R, T253V, and K264R), yielded the construct, Lsil ${ }_{\text {cryst }}$, used in the crystallographic studies described here. Among the seven mutations in the construct Lsi $1_{\text {cryst }}$, four mutations (C66A, T93V, C139A, and T253V) in TM helices enhanced the thermo-stability of Lsil in the detergent micelle. Three lysine residues (K50R, K232R, and $\mathrm{K} 264 \mathrm{R}$ ) in loop regions are mutated to arginine to reduce the surface entropy, hoping that mutants may improve the crystal packing.

Infected Sf 9 cells were harvested by centrifugation $(8000 \times g, 15 \mathrm{~min})$, and were disrupted by an ultrasonic disrupter UD-211 (TOMY). After centrifugation $(3000 \times g, 10 \mathrm{~min})$, the supernatant was ultra-centrifuged $(200,000 \times g, 1 \mathrm{~h})$, and membrane fraction was collected and homogenized. The crude membrane fractions were solubilized for $1 \mathrm{~h}$ in a buffer containing $500 \mathrm{mM} \mathrm{NaCl}, 20 \mathrm{mM}$ Tris- $\mathrm{HCl} \mathrm{pH}$ $8.0,6 \%(\mathrm{w} / \mathrm{v})$ glycerol, $1.8 \%(\mathrm{w} / \mathrm{v}) n$-dodecyl- $\beta$-D-maltopyranoside (DDM),
$0.06 \mathrm{mg} / \mathrm{ml}$ RNase A. Insoluble material was removed by ultracentrifugation $(148,500 \times g, 1.5 \mathrm{~h})$ and the supernatant was incubated with TALON cobalt affinity resin (Clontech) for $3 \mathrm{~h}$ in the presence of $10 \mathrm{mM}$ imidazole. After washing with a buffer containing $15 \mathrm{mM}$ imidazole, $500 \mathrm{mM} \mathrm{NaCl}, 20 \mathrm{mM}$ HEPES-NaOH pH 7.5, $10 \%(\mathrm{w} / \mathrm{v})$ glycerol, and $0.02 \%(\mathrm{w} / \mathrm{v}) \mathrm{DDM}$, Lsil mutants were eluted by application of a buffer containing $150 \mathrm{mM}$ imidazole, $500 \mathrm{mM} \mathrm{NaCl}, 20 \mathrm{mM}$ HEPES-NaOH pH 7.5, 10\% (w/v) glycerol, and $0.02 \%(w / v)$ DDM. The eluates were precipitated in the presence of $22.2 \%$ ( $\mathrm{w} / \mathrm{v}$ ) PEG 1500, and then dissolved in a buffer composed of $500 \mathrm{mM} \mathrm{NaCl}, 20 \mathrm{mM}$ HEPES-NaOH pH 7.5, 10\% (w/v) glycerol and $1 \%(\mathrm{w} / \mathrm{v}) n$-octyl- $\beta$-D-glucoside (OG). The octa-His tag was cleaved with hexa-His-tagged $\operatorname{TEV}_{\mathrm{SH}^{3}}{ }^{33}$ (3:1 mass ratio of Lsil to $\mathrm{TEV}_{\mathrm{SH}}$ ) overnight, and the protein was re-chromatographed on a TALON cobalt affinity resin. The tag cleaved Lsil was further purified by gel filtration (Superdex 200 Increase 10/300 GL column) in $500 \mathrm{mM} \mathrm{NaCl}, 20 \mathrm{mM}$ HEPES-NaOH pH 7.5, and 1\% (w/v) OG. All steps were performed at $4{ }^{\circ} \mathrm{C}$ unless otherwise noted.

Crystallization. The purified protein was concentrated to about $10 \mathrm{mg} / \mathrm{ml}$ using a $50 \mathrm{kD}$ molecular weight cut-off centrifugal filter device. The Lsil $1_{\text {cyst }}$ crystals were obtained at $7{ }^{\circ} \mathrm{C}$ by vapor diffusion sitting drop method by mixing $1: 1(\mathrm{v} / \mathrm{v})$ ratio of protein and a reservoir solution containing 39-50\% (w/v) PEG 400, $100 \mathrm{mM}$ Gly$\mathrm{NaOH} \mathrm{pH} 9.5,1 \%(\mathrm{w} / \mathrm{v}) \mathrm{OG}$, and $0.1 \%(\mathrm{w} / \mathrm{v})$ cholesteryl hemisuccinate (CHS). Both pyramidal and rod-shaped crystals appeared in the same crystallization drops, but the diffraction limit of the pyramidal crystals was around $7 \AA$ resolution. The rod-shaped crystals were collected and soaked in a solution containing $41 \%(\mathrm{w} / \mathrm{v})$ PEG 400, $500 \mathrm{mM} \mathrm{NaCl}, 20 \mathrm{mM}$ HEPES-NaOH pH 7.5, $100 \mathrm{mM}$ Bis-Tris HCl pH $7.0,2 \%(\mathrm{w} / \mathrm{v}) \mathrm{OG}, 0.1 \%(\mathrm{w} / \mathrm{v}) \mathrm{CHS}$, then flash-frozen in liquid nitrogen for X-ray diffraction experiment. Some crystals were soaked in a crystallization buffer supplemented with $44 \mathrm{mM} \mathrm{Si}(\mathrm{OH})_{4}$ or a saturated concentration of $\mathrm{Ge}(\mathrm{OH})_{4}$ for $5 \mathrm{~min}$ prior to freezing. A fresh $\mathrm{Si}(\mathrm{OH})_{4}$ solution was prepared to avoid the oligomerization of silicic acids.

Structure determination. X-ray diffraction data were collected at SPring-8 BL41XU or BL44XU and were processed with $\mathrm{XDS}^{34}$. The crystals belong to the space group $P 2_{1}$ (unit-cell parameters $a=89.5 \AA, b=91.4 \AA, c=166.1 \AA$, and $\beta=$ $102.1^{\circ}$. While some reflections remained sharp, the others were diffused with stronger intensities. This unusual pattern was induced by so-called lattice-translocation defects, in which two identical but translated lattices coexist as a single mosaic block in a crystal. We thus corrected the intensities based on the method reported previously ${ }^{16}$. In brief, if the intensity for each unit cell is $I_{\text {unit }}$, the total intensity is

$$
I_{\text {total }}=\left(2 \kappa^{2}-2 \kappa+1\right)\left[1+2 \kappa(1-\kappa) /\left(2 \kappa^{2}-2 \kappa+1\right) \cos \left(2 \pi \mathbf{h} \mathbf{t}_{\mathbf{d}}\right)\right] I_{\text {unit }}
$$

where the translocation vector $\mathbf{t}_{\mathbf{d}}$ and the fraction $\kappa$ were determined to be $(1 / 3,0$, $1 / 3$ ) and 0.30 , respectively. The corrected intensities gave significantly smaller values for R-factor and free R-factor. They were 0.343 for the R-factor and 0.365 for the free R-factor before the correction and 0.245 for the R-factor, and 0.273 for the free R-factor after the correction. The initial phase information was obtained by molecular replacement with Phaser ${ }^{35}$ using a homology model of Lsil as a search probe, created from the crystal structure of Archaeoglobus fulgidus AQP (PDB $3 \mathrm{NE} 2$ ). Two tetramers were manually built using $\mathrm{COOT}^{36}$ based on the electron density map calculated at a $1.8 \AA$ A resolution and refined using phenix.refine ${ }^{37}$. The two Lsil tetramers contained eight sodium ions, four OGs, two CHSs, and eleven PEGs. During the refinement process, coordinates, temperature factors, TLS were refined, and non-crystallographic symmetry restraints were not applied. The statistics for refinement was shown in Table 1. Figures were prepared using Cuemol2 (http://www.cuemol.org) or PyMOL (http://www.pymol.org), and the channel of Lsi1 $_{\text {cryst }}$ was analyzed using HOLE $2^{38}$.

Functional assay in Sf9 cells. The full-length Lsi1, including the octa-His tag and EGFP at the C-terminus of the Lsil construct (CE-Lsi1), was cloned into the pFastBacl vector. Similarly, codon-optimized GlpF, AQPM, and PfAQP genes were synthesized (Integrated DNA Technologies), amplified by PCR, cloned into the pFastBacl vector using a seamless cloning method. All mutated Lsi1 genes (e.g., Thr65 $5_{\mathrm{TM} 1}$ variants, CE-Lsi1_ $\Delta \mathrm{NC}$, and CE-Lsi1 $1_{\text {cryst }}$ ) used in the functional assay were generated using the QuikChange II site-directed mutagenesis method (Stratagene)

Sf9 cells infected with P3 viruses of interested CE-Lsil mutants or other AQPs (CE-GlpF, CE-AQPM, and CE-PfAQP) were grown at $27^{\circ} \mathrm{C}$ for $24 \mathrm{~h}$ after infection, followed by growing at $20^{\circ} \mathrm{C}$ for an additional $24 \mathrm{~h}$. Cell pellets were suspended with $900 \mu \mathrm{l}$ of PBS buffer $(200 \mathrm{mM} \mathrm{NaCl}, 2.68 \mathrm{mM} \mathrm{KCl}, 10 \mathrm{mM}$ $\mathrm{Na}_{2} \mathrm{HPO}_{4}$, and $2 \mathrm{mM} \mathrm{KH}_{2} \mathrm{PO}_{4}$ ), and then mixed with about $100 \mu \mathrm{l}$ of PBS buffer or PBS buffer containing $1 \mathrm{mM} \mathrm{Ge}(\mathrm{OH})_{4}$ or $100 \mu \mathrm{M} \mathrm{As}(\mathrm{OH})_{3}$. For one assay, $1.5 \times 10^{7}$ cells were used, and the suspension was in a total volume of $1 \mathrm{ml}$. After a subsequent $5 \mathrm{~min}$ incubation, cells were collected and then dried in a rotary evaporator for 2 days. After nitric acid digestion, the concentration of Ge or As of the dried Sf 9 cells were determined with ICP-MS (inductively coupled plasma-mass spectrometry 7700X; Agilent Technologies). The entire experiment was performed in triplicate. 
To determine the protein expression in the Sf9 cells, $2.0 \times 10^{6}$ cells were solubilized with $125 \mathrm{mM}$ Tris-HCl, pH 6.8, 4\% SDS, 20\% glycerol, $1.5 \mu \mathrm{M}$ aprotinin, $10 \mu \mathrm{M}$ leupeptin, $10 \mu \mathrm{g} / \mathrm{ml}$ trypsin inhibitor, and $1 \mathrm{mM}$ PMSF, and then sonicated. The protein contents were determined with a BCA protein assay kit (TaKaRa). A $20 \mu \mathrm{g}$ of total protein from each sample was separated by SDS-PAGE, transferred to PVDF membrane, detected with the anti-green fluorescent protein tag polyclonal antibody-HRP-DirecT (MBL). As a loading control, the solubilized cell was stained by $\mathrm{CBB}$.

Transport activity assay in Xenopus oocytes. Oocytes for transport activity assay were isolated from $X$. laevis. Procedures for defolliculation, culture conditions, and selection were the same as described previously ${ }^{6}$. The ORFs of native and mutated $L s i 1$, and GlpF were amplified from pFastbacl plasmids containing the genes described above by PCR. The ORFs of these genes were inserted into the BglII site of a Xenopus oocyte expression vector, pX $\beta$ G-ev1 with FLAG tag (DYKDDDDK). Capped RNA was synthesized by in vitro transcription with a mMESSAGE mMACHINE High Yield Capped RNA Transcription Kit (Ambion). A volume of $50 \mathrm{nl}\left(1 \mathrm{ng} \mathrm{nl}^{-1}\right)$ cRNA or RNase-free water as a negative control was injected into the oocyte. After $1-3$ days of incubation in Modified Barth's Saline (MBS) at $18^{\circ} \mathrm{C}$, oocytes were subjected to the transport activity assay.

To determine the protein expression in the oocytes, membrane protein was collected from the oocytes by centrifugation according to the previous report ${ }^{10}$. A $5 \mu \mathrm{g}$ of a membrane protein from each sample was separated by SDS-PAGE, transferred to PVDF membrane, probed with the anti-DYKDDDDK tag monoclonal antibody (Invitrogen), and detected with Anti-Mouse IgG HRP Conjugate (Promega) or mouse monoclonal ANTI-FLAG ${ }^{\circledR}$ M2-HRP antibody (Sigma-Aldrich). As a loading control, the membrane was stained by CBB.

For Ge and As transport activity determination, oocytes were incubated in MBS with $1 \mathrm{mM} \mathrm{Ge}(\mathrm{OH})_{4}$ or $100 \mu \mathrm{M} \mathrm{As}(\mathrm{OH})_{3}$ for $30 \mathrm{~min}$ at $18^{\circ} \mathrm{C}$. At the end of the uptake, the oocytes were washed in ice-cold MBS and digested with $\mathrm{HNO}_{3}$. Ge and As concentrations in the digested solution were determined by ICP-MS as described above. The permeability of oocytes for glycerol and water was determined by a swelling assay. After cRNA injection and initial incubation in control MBS for 2-3 days, oocytes were transferred to a five-fold diluted MBS for water permeability assay. Changes in the oocyte volume were monitored within $180 \mathrm{~s}$ at $20 \mathrm{~s}$ intervals. For the glycerol permeability, oocytes were transferred to an isotonic solution containing five-fold diluted MBS supplemented with glycerol to adjust the osmolarity (glycerol concentration was $170 \mathrm{mM}$ ). Changes in the oocyte volume were recorded as described above. Permeability of glycerol and water was presented as oocyte volume change $\left[\mathrm{d}\left(V V_{0}^{-1}\right) \mathrm{d} t^{-1}\right]$. In this study, we used two systems (Xenopus oocytes and insect cells) for transport analysis, and the functional results obtained were equivalent or similar in either system.

QM/MM calculation. We placed silicic acid molecules (The Cambridge Crystallographic Data Center, the deposition number 1406687) by hand so that their oxygen atoms overlapping with water molecules in the crystal structure. This process allowed us to build eight $\mathrm{Si}$ molecules ( $\mathrm{Si} 1-\mathrm{Si}$ ), and water molecules employed for the modeling were shown in Fig. 6e. Theoretical analysis (QM/MM calculations and the MD simulation) for Lsil was performed using the X-ray crystal structure. Hydrogen atoms were generated and energetically optimized with the CHARMM program ${ }^{39}$, while the positions of all non-hydrogen atoms were fixed, and all titratable groups were maintained in their standard protonation state at $\mathrm{pH}$ 7. We added additional counterions to neutralize the entire system. Atomic partial charges of the amino acid were adopted from the all-atom CHARMM22 parameter set ${ }^{40}$

For QM/MM calculations, we used the Qsite ${ }^{41}$ code and employed the restricted density functional-theory method with the B3LYP functional and LACVP* basis sets. The geometries were refined by constrained QM/MM optimization. To avoid the uncertainty associated with the MM force field, we constrained most of the atoms in the surrounding MM region. Namely, the coordinates of the heavy atoms in the MM region were fixed to the original X-ray coordinates. In contrast, those of the $\mathrm{H}$ atoms in the MM region were optimized using the OPLS2005 force field. All atomic coordinates in the QM region were fully relaxed (i.e., not fixed) in the QM/ $\mathrm{MM}$ calculations. The $\mathrm{QM}$ region was defined as the modeled $\mathrm{Si}(\mathrm{OH})_{4}$ in the channel (Si1-Si8; Fig. 6d), water molecules (Fig. 6c) in the channel (Fig. 6), and amino acids H-bonded with them (Thr65, Gln84, Gly104, Val203, Ser207, Gly215, Gly216, the sidechain of Asn108, Met177, Thr181, Asn219, and Arg222, and the backbone of His106, Thr156, and Ser217)

Molecular dynamics simulation. For the MD simulation, the Lsil tetramer was embedded in a lipid bilayer consisting of 314 1-palmitoyl-2-oleyl-sn-glycero-3phosphocholine (POPC), using the CHARMM-GUI program ${ }^{42}$. Then the system was soaked in $385 \mathrm{Si}(\mathrm{OH})_{4}$ and 24215 TIP3P water models ${ }^{43}\left(\sim 1 \mathrm{M}\right.$ of $\left.\mathrm{Si}(\mathrm{OH})_{4}\right)$. After structural optimization with positional restraints on heavy atoms of the Lsi1 tetramer, the system was heated from 0.1 to $300 \mathrm{~K}$ over $5.5 \mathrm{ps}$ with a time step of $0.01 \mathrm{fs}$, equilibrated at $300 \mathrm{~K}$ for $1 \mathrm{~ns}$ with a time step of $0.5 \mathrm{fs}$, and annealed from 300 to $0 \mathrm{~K}$ over $5.5 \mathrm{ps}$ with a time step of $0.01 \mathrm{fs}$. The same procedure was repeated with positional restraints on the heavy atom of the protein backbone. The same procedure was repeated without positional restraints on any atoms. After an equilibrating MD run for $15 \mathrm{~ns}$, a production run was conducted over $450 \mathrm{~ns}$ with a time step of $1.5 \mathrm{fs}$. The SHAKE algorithm was used for hydrogen constraints ${ }^{44}$. The MD simulation was based on the CHARMM force field for protein residues ${ }^{40}$ and lipids ${ }^{45}$. For $\mathrm{Si}(\mathrm{OH})_{4}$, we employed the parameter set reported by Piane et al. ${ }^{46}$, except for the parameter set for the $\mathrm{O}-\mathrm{H}$ bond, which was taken from the generalized Amber force field $(\mathrm{GAFF})^{47}$. The atomic partial charges of $\mathrm{Si}(\mathrm{OH})_{4}$ were determined by fitting the electrostatic potential by using the RESP procedure ${ }^{48}$. They were 1.0631 for $\mathrm{Si}$ atom, -0.7103 for $\mathrm{O}$ atom, and 0.4445 for $\mathrm{H}$ atom, respectively. The electronic wave functions were calculated after geometry optimization with the density functional theory of the B3LYP/6-31G** level by using JAGUAR $^{49}$. The MD simulation was conducted using the MD engine NAMD ${ }^{50}$.

Reporting summary. Further information on research design is available in the Nature Research Reporting Summary linked to this article.

\section{Data availability}

The coordinates and structure factors for Lsil $1_{\text {cryst }}$ have been deposited in the Protein Data Bank (PDB) with accession number 7CJS. The source data for Figs. 2, 5, and 7, and Supplementary Figs. 3, 6-8, 10-12, and 16 have been provided as the Source Data file. Any other data associated with this manuscript are available from the authors at a reasonable request. Source data are provided with this paper.

Received: 24 September 2020; Accepted: 7 October 2021; Published online: 29 October 2021

\section{References}

1. Mitani-Ueno, N. \& Ma, J. F. Linking transport system of silicon with its accumulation in different plant species. Soil Sci. Plant Nutr. 67, 10-17 (2021)

2. Ma, J. F. Role of silicon in enhancing the resistance of plants to biotic and abiotic stresses. Soil Sci. Plant Nutr. 50, 11-18 (2004).

3. Ma, J. F. \& Takahashi, E. Soil, Fertilizer, and Plant Silicon Research in Japan 1st edn (Elsevier, 2002).

4. Savant, N. K., Snyder, G. H. \& Datnoff, L. E. Silicon management and sustainable rice production. Adv. Agron. 58, 151-199 (1997).

5. Ma, J. F. \& Yamaji, N. A cooperative system of silicon transport in plants. Trends Plant Sci. 20, 435-442 (2015).

6. Ma, J. F. et al. A silicon transporter in rice. Nature 440, 688-691 (2006)

7. Ma, J. F. et al. An efflux transporter of silicon in rice. Nature 448, 209-212 (2007).

8. Wallace, I. S., Choi, W. G. \& Roberts, D. M. The structure, function, and regulation of the nodulin 26-like intrinsic protein family of plant aquaglyceroporins. Biochim. Biophys. Acta 1758, 1165-1175 (2006).

9. Wallace, I. S. \& Roberts, D. M. Distinct transport selectivity of two structural subclasses of the nodulin-like intrinsic protein family of plant aquaglyceroporin channels. Biochemistry 44, 16826-16834 (2005).

10. Mitani-Ueno, N., Yamaji, N., Zhao, F. J. \& Ma, J. F. The aromatic/arginine selectivity filter of NIP aquaporins plays a critical role in substrate selectivity for silicon, boron, and arsenic. J. Exp. Bot. 62, 4391-4398 (2011).

11. Ma, J. F. et al. Transporters of arsenite in rice and their role in arsenic accumulation in rice grain. Proc. Natl Acad. Sci. USA 105, 9931-9935 (2008).

12. Pommerrenig, B., Diehn, T. A. \& Bienert, G. P. Metalloido-porins: essentiality of Nodulin 26-like intrinsic proteins in metalloid transport. Plant Sci. 238, 212-227 (2015)

13. Sui, H., Han, B. G., Lee, J. K., Walian, P. \& Jap, B. K. Structural basis of waterspecific transport through the AQP1 water channel. Nature 414, 872-878 (2001).

14. Fu, D. et al. Structure of a glycerol-conducting channel and the basis for its selectivity. Science 290, 481-486 (2000).

15. Kawate, T. \& Gouaux, E. Fluorescence-detection size-exclusion chromatography for precrystallization screening of integral membrane proteins. Structure 14, 673-681 (2006)

16. Wang, J., Kamtekar, S., Berman, A. J. \& Steitz, T. A. Correction of X-ray intensities from single crystals containing lattice-translocation defects. Acta Crystallogr. Sect. D., Biol. Crystallogr. 61, 67-74 (2005).

17. Tornroth-Horsefield, S. et al. Structural mechanism of plant aquaporin gating. Nature 439, 688-694 (2006)

18. Kirscht, A. et al. Crystal structure of an ammonia-permeable aquaporin. PLoS Biol. 14, e1002411 (2016).

19. Murata, K. et al. Structural determinants of water permeation through aquaporin-1. Nature 407, 599-605 (2000).

20. Gonen, T. \& Walz, T. The structure of aquaporins. Q Rev. Biophys. 39, 361-396 (2006)

21. Eriksson, U. K. et al. Subangstrom resolution $\mathrm{x}$-ray structure details aquaporin-water interactions. Science 340, 1346-1349 (2013). 
22. Tajkhorshid, E. et al. Control of the selectivity of the aquaporin water channel family by global orientational tuning. Science 296, 525-530 (2002).

23. de Groot, B. L. \& Grubmuller, H. Water permeation across biological membranes: mechanism and dynamics of aquaporin-1 and GlpF. Science 294, 2353-2357 (2001).

24. Newby, Z. E. et al. Crystal structure of the aquaglyceroporin PfAQP from the malarial parasite Plasmodium falciparum. Nat. Struct. Mol. Biol. 15, 619-625 (2008).

25. Lee, J. K. et al. Structural basis for conductance by the archaeal aquaporin AqpM at 1.68 A. Proc. Natl Acad. Sci. USA 102, 18932-18937 (2005).

26. Deshmukh, R. K. et al. A precise spacing between the NPA domains of aquaporins is essential for silicon permeability in plants. Plant J. 83, 489-500 (2015).

27. Sun, H. et al. Tomato roots have a functional silicon influx transporter but not a functional silicon efflux transporter. Plant Cell Environ. 43, 732-744 (2020).

28. Abascal, F., Irisarri, I. \& Zardoya, R. Diversity and evolution of membrane intrinsic proteins. Biochim. Biophys. Acta 1840, 1468-1481 (2014).

29. Gregoire, C. et al. Discovery of a multigene family of aquaporin silicon transporters in the primitive plant Equisetum arvense. Plant J. 72, 320-330 (2012).

30. Garneau, A. P. et al. Aquaporins mediate silicon transport in humans. PLoS One 10, e0136149 (2015).

31. Gotfryd, K. et al. Human adipose glycerol flux is regulated by a $\mathrm{pH}$ gate in AQP10. Nat. Commun. 9, 4749 (2018).

32. Doyle, D. A. et al. The structure of the potassium channel: molecular basis of $\mathrm{K}+$ conduction and selectivity. Science 280, 69-77 (1998).

33. van den Berg, S., Lofdahl, P. A., Hard, T. \& Berglund, H. Improved solubility of TEV protease by directed evolution. J. Biotechnol. 121, 291-298 (2006).

34. Kabsch, W. XDS. Acta Crystallogr. Sect. D., Biol. Crystallogr. 66, 125-132 (2010).

35. McCoy, A. J. et al. Phaser crystallographic software. J. Appl. Crystallogr. 40 658-674 (2007).

36. Emsley, P. \& Cowtan, K. Coot: model-building tools for molecular graphics. Acta Crystallogr. Sect. D., Biol. Crystallogr. 60, 2126-2132 (2004).

37. Adams, P. D. et al. PHENIX: a comprehensive Python-based system for macromolecular structure solution. Acta Crystallogr. Sect. D., Biol. Crystallogr. 66, 213-221 (2010).

38. Smart, O. S., Neduvelil, J. G., Wang, X., Wallace, B. A. \& Sansom, M. S. P. HOLE: A program for the analysis of the pore dimensions of ion channel structural models. J. Mol. Graph Model 14, 354-360 (1996).

39. Miller, B. T. et al. CHARMMing: a new, flexible web portal for CHARMM. J. Chem. Inf. Model 48, 1920-1929 (2008).

40. Best, R. B. et al. Optimization of the additive CHARMM all-atom protein force field targeting improved sampling of the backbone phi, psi and sidechain chi(1) and chi(2) dihedral angles. J. Chem. Theory Comput. 8, 3257-3273 (2012).

41. QSite (Schrödinger, LLC, 2012).

42. Jo, S., Kim, T., Iyer, V. G. \& Im, W. CHARMM-GUI: a web-based graphical user interface for CHARMM. J. Comput. Chem. 29, 1859-1865 (2008).

43. Jorgensen, W. L., Chandrasekhar, J., Madura, J. D., Impey, R. W. \& Klein, M. L. Comparison of simple potential functions for simulating liquid water. J. Chem. Phys. 79, 926-935 (1983).

44. Ryckaert, J. P., Ciccotti, G. \& Berendsen, H. J. C. Numerical-integration of Cartesian equations of motion of a system with constraints-moleculardynamics of N-alkanes. J. Comput. Phys. 23, 327-341 (1977).

45. Klauda, J. B. et al. Update of the CHARMM all-atom additive force field for lipids: validation on six lipid types. J. Phys. Chem. B 114, 7830-7843 (2010).

46. Piane, M. D., Potthoff, S., Brinker, C. J. \& Colombi, L. C. Molecular dynamics simulations of the silica-cell membrane interaction: insights on biomineralization and nanotoxicity. J. Phys. Chem. C 122, 21330-21343 (2018).

47. Wang, J., Wolf, R. M., Caldwell, J. W., Kollman, P. A. \& Case, D. A. Development and testing of a general amber force field. J. Comput. Chem. $\mathbf{2 5}$, 1157-1174 (2004).

48. Bayly, C. I., Cieplak, P., Cornell, W. D. \& Kollman, P. A. A well-behaved electrostatic potential based method using charge restraints for deriving atomic charges-the Resp model. J. Phys. Chem. 97, 10269-10280 (1993).

49. Jaguar, version 8.0 (Schrödinger, LLC, 2013).
50. Phillips, J. C. et al. Scalable molecular dynamics with NAMD. J. Comput. Chem. 26, 1781-1802 (2005).

51. Kumar, S., Stecher, G., Li, M., Knyaz, C. \& Tamura, K. MEGA X: molecular evolutionary genetics analysis across computing platforms. Mol. Biol. Evol. 35 $1547-1549$ (2018).

52. Sievers, F. \& Higgins, D. G. Clustal Omega, accurate alignment of very large numbers of sequences. Methods Mol. Biol. 1079, 105-116 (2014).

\section{Acknowledgements}

This work was supported by JSPS KAKENHI Grants JP16H06296 (M.S. and J.F.M.), JP21H05034 (M.S. and J.F.M.), JP17H06879 (Y.S.), JP19K16056 (Y.S.), 18H05155 (H.I.), $18 \mathrm{H} 01937$ (H.I.), $20 \mathrm{H} 03217$ (H.I.), $20 \mathrm{H} 05090$ (H.I.), 16H06560 (K.S.) and $18 \mathrm{H} 01186$ (K.S.). This work was also supported by JST CREST JPMJCR1656 (H.I.) and the Interdisciplinary Computational Science Program in CCS, University of Tsukuba. The X-ray diffraction experiment was performed at beamlines 41XU and 44XU of SPring-8 (Hyogo, Japan) with the approval of the Japan Synchrotron Radiation Research Institute (JASRI) (proposals 2016B6621, 2017A6724, 2017B6724, 2018A2530, 2018B2530, 2019A2559 and 2019B2559), and we thank the staff at SPring-8 for their help. We thank S. Yonekura for the discussion, A. Morita, Y. Takahashi, M. Hikasa and S. Rikiishi for experimental assistance.

\section{Author contributions}

M.S. and J.F.M. conceived the project; M.S. and K.M. screened genes by FSEC; Y.S., K.M., L.Y., J.-R.S. and M.S. purified Lsil. Y.S. and K.M. crystallized Lsi1; M.S. and Y.S. determined the Lsi1 structure. Y.S., N.M.-U., K.M., N.Y., S.H., M.S., and J.F.M. performed functional assays; K.S. and H.I. performed theoretical calculations; Y.S., M.S., N.M.-U. and J.F.M. wrote the paper with inputs from all authors.

\section{Competing interests}

The authors declare no competing interests.

\section{Additional information}

Supplementary information The online version contains supplementary material available at https://doi.org/10.1038/s41467-021-26535-x.

Correspondence and requests for materials should be addressed to Jian Feng Ma or Michihiro Suga.

Peer review information Nature Communications thanks Susanna Törnroth Horsefield, Hai Lin, and the other, anonymous, reviewer(s) for their contribution to the peer review of this work. Peer reviewer reports are available.

Reprints and permission information is available at http://www.nature.com/reprints

Publisher's note Springer Nature remains neutral with regard to jurisdictional claims in published maps and institutional affiliations.

Open Access This article is licensed under a Creative Commons Attribution 4.0 International License, which permits use, sharing, adaptation, distribution and reproduction in any medium or format, as long as you give appropriate credit to the original author(s) and the source, provide a link to the Creative Commons license, and indicate if changes were made. The images or other third party material in this article are included in the article's Creative Commons license, unless indicated otherwise in a credit line to the material. If material is not included in the article's Creative Commons license and your intended use is not permitted by statutory regulation or exceeds the permitted use, you will need to obtain permission directly from the copyright holder. To view a copy of this license, visit http://creativecommons.org/ licenses/by/4.0/.

(c) The Author(s) 2021 